\title{
HOMOGENIZATION AND DIFFUSION ASYMPTOTICS OF THE LINEAR BOLTZMANN EQUATION
}

\author{
Thierry Goudon ${ }^{1,2}$ And Antoine Mellet ${ }^{3}$
}

\begin{abstract}
We investigate the diffusion limit for general conservative Boltzmann equations with oscillating coefficients. Oscillations have a frequency of the same order as the inverse of the mean free path, and the coefficients may depend on both slow and fast variables. Passing to the limit, we are led to an effective drift-diffusion equation. We also describe the diffusive behaviour when the equilibrium function has a non-vanishing flux.
\end{abstract}

Mathematics Subject Classification. 35Q35, 82C70, 76P05, 74Q99, 35B27.

Received September 30, 2002. Revised March 4, 2003.

\section{INTRODUCTION}

In this paper, we investigate the asymptotic behaviour as $\varepsilon$ goes to zero of the solution of the following kinetic equation:

$$
\begin{cases}\varepsilon \partial_{t} f_{\varepsilon}+a(v) \cdot \nabla_{x} f_{\varepsilon}=\frac{1}{\varepsilon} Q_{\varepsilon}\left(f_{\varepsilon}\right) & \text { in }[0, \infty) \times \mathbb{R}^{N} \times V, \\ f_{\varepsilon}(0, x, v)=f_{\varepsilon}^{0}(x, v) & \text { in } \mathbb{R}^{N} \times V .\end{cases}
$$

Such a problem naturally arises when modeling the behaviour of a cloud of "particles" (e.g., electrons moving in a semiconductor material or neutrons moving in a nuclear reactor). The unknown $f_{\varepsilon}(t, x, v) \geq 0$ can be interpreted as the density of particles occupying the position $x$ with a physical state described by the variable $v \in V$ at time $t \geq 0$. Usually $v$ is nothing but the translation velocity of the particle; more complicated modeling can be handled. The set $V$ is endowed with a measure $\mathrm{d} \mu$ and the quantity $\int_{\Omega} \int_{\mathcal{V}} f_{\varepsilon} \mathrm{d} \mu(v) \mathrm{d} x$ is the number of particles at time $t$ in the domain $\Omega \times \mathcal{V}$ of the phase space. Later, we will make precise the crucial properties required on $(V, \mathrm{~d} \mu)$. The left-hand side in (1) describes the transport of the particles, with a velocity field $a: V \longrightarrow \mathbb{R}^{N}$, while the right-hand side takes into account the interactions that particles may undergo while crossing the device.

The parameter $\varepsilon$, which tends to zero, is related to the following physical scaling:

- comparing the mean free path of the particles, $\lambda$, and a typical length of heterogeneities of the medium, $\ell$, with an observation length scale $L$, we assume that the ratio $\ell / L$ and $\lambda / L$ have the same order $\varepsilon \ll 1$;

- we observe the system at a large time scale, of order $1 / \varepsilon \gg 1$.

Keywords and phrases. Boltzmann equation, diffusion approximation, homogenization, drift-diffusion equation.

1 CNRS, Université des Sciences et Technologies Lille 1, UFR Mathématiques Pures et Appliquées, Cité Scientifique,

59655 Villeneuve-d'Ascq Cedex, France; e-mail: thierry.goudon@univ-lille1.fr

2 INRIA-Sophia, Project Caiman.

3 Mathématiques pour l'Industrie et la Physique, UMR 5640, Université Paul Sabatier, 118 route de Narbonne,

31062 Toulouse Cedex, France; e-mail: mellet@mip.ups-tlse.fr

(c) EDP Sciences, SMAI 2003 
Hence, the $\varepsilon$ in front of $\partial_{t}$ is related to the long time scaling, while the $1 / \varepsilon$ in front of the collision operator means that particles undergo more and more interactions. These interactions modify the physical state of the particles, but are localized in time and space. They can be described by the following integral operator:

$$
Q_{\varepsilon}(f)(x, v)=\int_{V} \sigma\left(x, x / \varepsilon, v, v^{\prime}\right) f\left(v^{\prime}\right) \mathrm{d} \mu\left(v^{\prime}\right)-\Sigma(x, x / \varepsilon, v) f(v) .
$$

Note that this operator depends on $\varepsilon$ through the oscillating variable $x / \varepsilon$, which takes into account heterogeneities of the medium. These heterogeneities will be assumed periodic. We therefore suppose that $y \longmapsto \sigma\left(x, y, v, v^{\prime}\right)$ is $Y$-periodic, with $Y=(0,1)^{N}$. Moreover, assuming that the total density is conserved:

$$
\int_{\mathbb{R}^{N}} \int_{V} f_{\varepsilon}(t) \mathrm{d} \mu(v) \mathrm{d} x=\int_{\mathbb{R}^{N}} \int_{V} f_{\varepsilon}^{0} \mathrm{~d} \mu(v) \mathrm{d} x
$$

we are led to the following relation:

$$
\Sigma(x, x / \varepsilon, v)=\int_{V} \sigma\left(x, x / \varepsilon, v^{\prime}, v\right) \mathrm{d} \mu\left(v^{\prime}\right) .
$$

For a general introduction of the physical background, we refer the reader to the classical book of Cercignani [13]. The question of diffusion approximation of kinetic processes (limit $\lambda / L \ll 1$ and $\ell / L \sim 1$ ) has motivated a lot of works, with various fields of applications: neutron transport by Larsen and Keller [35], Rosseland approximation for radiative transfer problems by Bardos et al. [7] and Bardos et al. [8], discrete-velocity models by Lions and Toscani [37], semiconductors Boltzmann equations by Poupaud [40], semiconductors Boltzmann equations with Pauli exclusion principle by Golse and Poupaud [26] and Goudon and Mellet [28], general linear equation without detailed balance relation by Degond et al. [17], chemotaxis phenomena by Chalub et al. [14]... On the other hand, we can search for effective transport coefficients (limit $\ell / L \ll 1$ and $\lambda / L \sim 1$ ), as in Dumas and Golse [19] and Gérard and Golse [23]. In our case, the ordering assumes that these length scales have the same order. The problem is particularly relevant in neutron engineering (see Wigner [43]). A lot of progress has been made since the pionneering works of Larsen [33,34], Larsen and Williams [36], and Bensoussan et al. [9]. A Hilbertexpansion-based method has been successfully used by Goudon and Poupaud [29] and Goudon and Mellet [27], but in these papers, a crucial assumption is made that the equilibrium functions, i.e. solutions of $Q(f)=0$, do not depend on the fast variable $x / \varepsilon$. In this paper, we aim at filling that gap, by considering general oscillating kernels with modulated oscillations. On the other hand, the specific (and delicate) difficulties of the spectral problem have been treated by Allaire and Bal [2], for the case of purely oscillating coefficients, by using a factorization method (inspired from nuclear engineering). For the eigenvalue problem, the spatial modulations have very complicated concentration effects; they are investigated in the recent paper of Allaire et al. [3].

The paper is organized as follows. In Section 2, the limit equation is guessed by means of a formal doublescale series expansion. At this formal level, we realize that the result depends on the solvability of certain cell problems, with variables $y, v$, parametrized by $x$. We solve these auxilliary equations by applying the Fredholm alternative. This is done in Section 3, where assumptions on the set $V$, the cross-section $\sigma$, the initial data $f_{\varepsilon}^{0}$ are precisely stated, and we also give the complete statement of our main convergence result. However, solvability of the cell problems depends also on the condition that the flux associated to the equilibrium state vanishes. We justify the convergence result with this condition in Section 4. Then, in Section 5, we investigate the behaviour of the solutions of (1) when this null flux condition is not fulfilled. (For the spectral problem, we refer to this respect to Bal [6] and Capdeboscq [11].) In view of the natural conservation (3), the only immediate estimate of $f_{\varepsilon}$ is in $\mathrm{L}^{1}\left(\mathbb{R}^{N} \times V\right)$. Therefore, we can expect only convergence in a very weak sense (vague convergence for measures), and the limit is, a priori, only a measure. Hence, one of the main contribution of the paper consists of treating the asymptotic regime having only this physical estimate. Then, we detail in Section 6 some dissipative properties which allow us, in some situations, to improve the regularity of the limit. 


\section{Formal Asymptotics}

In this section, we formally investigate the asymptotic behaviour of $f^{\varepsilon}$ by introducing the following formal double-scale expansion of $f_{\varepsilon}$ :

$$
f_{\varepsilon}(t, x, v)=f^{0}(t, x, x / \varepsilon, v)+\varepsilon f^{1}(t, x, x / \varepsilon, v)+\varepsilon^{2} f^{2}(t, x, x / \varepsilon, v)+\cdots
$$

where the functions $f^{i}(t, x, y, v)$ are $Y$-periodic with respect to $y$ for all $i$. We now insert this expansion in equation (1) remarking that

$$
\partial_{x}\left(f^{i}(t, x, x / \varepsilon, v)\right)=\left(\partial_{x} f^{i}\right)(t, x, x / \varepsilon, v)+\frac{1}{\varepsilon}\left(\partial_{y} f^{i}\right)(t, x, x / \varepsilon, v) .
$$

Identifying terms having the same power with respect to $\varepsilon$, we obtain the following set of cell equations, in which the $x$ variable is nothing but a parameter:

$$
\begin{cases}a(v) \cdot \nabla_{y} f^{0}-Q\left(f^{0}\right)=0 & \left(\varepsilon^{-1} \text { terms }\right), \\ a(v) \cdot \nabla_{y} f^{1}-Q\left(f^{1}\right)=-a(v) \cdot \nabla_{x} f^{0} & \left(\varepsilon^{0} \text { terms }\right), \\ a(v) \cdot \nabla_{y} f^{2}-Q\left(f^{2}\right)=-\partial_{t} f^{0}-a(v) \cdot \nabla_{x} f^{1} & \left(\varepsilon^{1} \text { terms }\right)\end{cases}
$$

where $Q$ stands for

$$
Q(f)=\int_{V} \sigma\left(x, y, v, v^{\prime}\right) f\left(v^{\prime}\right)-\sigma\left(x, y, v^{\prime}, v\right) f(v) \mathrm{d} \mu\left(v^{\prime}\right)
$$

(In the sequel, we shall note $Q_{\varepsilon}(x)=Q(x, x / \varepsilon)$.) In view of $(5)$, we introduce the following operator:

$$
\mathcal{T}=a(v) \cdot \nabla_{y}-Q
$$

with domain

$$
\mathcal{D}(\mathcal{T})=\left\{f \in \mathrm{L}^{p}(Y \times V), \text { such that } a(v) \cdot \nabla_{y} f \in \mathrm{L}^{p}(Y \times V)\right\}
$$

Then, the $\varepsilon^{-2}$ equation reads $f^{0} \in \operatorname{Ker}(\mathcal{T})$, and the other equations have the general form $\mathcal{T}(f)=g$. Therefore, the properties of the operator $\mathcal{T}$ will play a key role in the derivation of the asymptotic model. In particular, as a consequence of periodicity and conservation property of the operator $Q$, we remark that $\int_{Y} \int_{V} g \mathrm{~d} \mu(v) \mathrm{d} y=0$ is a necessary condition to solve $\mathcal{T}(f)=g$. We shall see later on that this condition is also sufficient (as a consequence of the Fredholm alternative for the operator $\mathcal{T}$ ). For the time being, let us assume the following facts:

(a) the kernel of $\mathcal{T}$ is spanned by a unique normalized and nonnegative function $F(x, y, v)$ :

$$
\operatorname{Ker}(\mathcal{T})=\operatorname{Span}\{F\}, \quad \int_{Y} \int_{V} F \mathrm{~d} \mu(v) \mathrm{d} y=1, \quad F(x, y, v) \geq 0
$$

(b) the equation $\mathcal{T}(f)=g$ has a solution (unique up to elements of $\operatorname{Ker}(\mathcal{T})$ ) if and only if $\int_{Y} \int_{V} g \mathrm{~d} \mu(v) \mathrm{d} y=0$

From (a), we deduce that $f^{0}(t, x, y, v)=\rho(t, x) F(x, y, v)$. Then, the $\varepsilon^{-1}$ equation becomes (applying formally the chain rule)

$$
\mathcal{T}\left(f^{1}\right)=-a(v) F(x, y, v) \cdot \nabla_{x} \rho(t, x)-\rho(t, x) a(v) \cdot \nabla_{x} F(x, y, v),
$$

the solvability of which, in view of (b), requires that the flux associated to the equilibrium function $F$ vanishes:

$$
\int_{Y} \int_{V} a(v) F(x, y, v) \mathrm{d} \mu(v) \mathrm{d} y=0 .
$$


When (6) holds, using (b) we can define the vector valued function $\chi(x, y, v)$ and the scalar function $\lambda(x, y, v)$ defined by the cell equations

$$
\begin{aligned}
\mathcal{T}\left(\chi_{i}\right) & =-a(v)_{i} F(x, y, v), \quad \forall i \in\{1, \ldots, N\} \\
\mathcal{T}(\lambda) & =-a(v) \cdot \nabla_{x} F(x, y, v) .
\end{aligned}
$$

We deduce:

$$
f^{1}(t, x, y, v)=\chi(x, y, v) \cdot \nabla_{x} \rho(t, x)+\lambda(x, y, v) \rho(t, x)+q(t, x) F(x, y, v) .
$$

Inserting this relation in the $\varepsilon^{0}$ equation, the solvability condition in (b) leads to the limit equation for $\rho(t, x)$ :

$$
\begin{aligned}
0 & =\partial_{t}\left(\int_{Y} \int_{V} f^{0} \mathrm{~d} \mu(v) \mathrm{d} y\right)+\nabla_{x} \cdot\left(\int_{Y} \int_{V} a(v) f^{1} \mathrm{~d} \mu(v) \mathrm{d} y\right) \\
& =\partial_{t} \rho+\nabla_{x} \cdot\left(\int_{Y} \int_{V} a(v) \otimes \chi \mathrm{d} \mu(v) \mathrm{d} y \nabla_{x} \rho+\int_{Y} \int_{V} a(v) \lambda \mathrm{d} \mu(v) \mathrm{d} y \rho\right) .
\end{aligned}
$$

(Note that, due to (6), the term $q(t, x) F$ in the definition of $f^{1}$ does not give any contribution in this formula.) Therefore, the density $\rho(t, x)$ satisfies the drift-diffusion equation

$$
\partial_{t} \rho-\operatorname{div}_{x}\left(D(x) \nabla_{x} \rho\right)+\operatorname{div}_{x}(U(x) \rho)=0
$$

with effective coefficients

$$
\left\{\begin{array}{l}
D(x)=-\int_{Y} \int_{V} a(v) \otimes \chi(x, y, v) \mathrm{d} \mu(v) \mathrm{d} y, \\
U(x)=\int_{Y} \int_{V} a(v) \lambda(x, y, v) \mathrm{d} \mu(v) \mathrm{d} y .
\end{array}\right.
$$

(We remark that the drift is due to the dependence of the cross section $\sigma$ with respect to the slow space variable, when solving the auxilliary problem (8).)

From a physical point of view, we can explain the limit as follows: in view of the conservation property of the operator, the macroscopic density

$$
\rho_{\varepsilon}(t, x)=\int_{V} f_{\varepsilon} \mathrm{d} \mu(v)
$$

and the macroscopic current

$$
J_{\varepsilon}(t, x)=\int_{V} \frac{a(v)}{\varepsilon} f_{\varepsilon} \mathrm{d} \mu(v)
$$

are related by the conservation equation

$$
\partial_{t} \rho_{\varepsilon}+\operatorname{div}_{x} J_{\varepsilon}=0 .
$$

When $\varepsilon$ goes to zero, we expect to justify that $\rho_{\varepsilon} \rightarrow \rho$ and $J_{\varepsilon} \rightarrow J$ (at least in some weak sense) with a limit current related to the density by a generalized Fick relation

$$
J=-D \nabla_{x} \rho+U \rho .
$$

We will come back to this approach in Section 6. This problem has been studied under some restrictive hypotheses (by using different strategies of proof): in [2] the cross-section does not depend on the slow variable, while in [29] and [27] the equilibrium function does not depend on the fast variable. The general situation is dealt with, for the spectral problem, in [3]. On the other hand, the reasoning depends crucially on the realization of the vanishing flux condition (6). The situation where this condition does not hold has been investigated for the delicate case of the eigenvalue problem in [6], and in [11] for diffusion equations. We will come back to this question in Section 5. 


\section{MAin RESUlts}

The remainder of the paper is devoted to the rigorous proof of the formal result obtained in the previous section: the convergence of $\rho_{\varepsilon}=\int_{V} f_{\varepsilon} \mathrm{d} \mu(v)$ to $\rho$, the solution of $(9)$. We split this section into five parts. First, we collect the various assumptions needed on the data of the problems, with some comments. Second, we study the cell problems described above and we discuss the realization of (a) and (b). Third, we discuss the properties of the effective coefficients; in particular positivity of the (symmetric part of the) matrix $D$. In the fourth part we set up some notations and compactness results. Finally, we give the statement of the convergence result.

\subsection{General assumptions}

First of all, let us make precise the nature of the measured space $(V, \mathrm{~d} \mu)$ :

$$
\text { (H1) }\left\{\begin{array}{l}
V \text { is a compact subset of } \mathbb{R}^{M} \text {, and the measure } \mu \text { satisfies } \mu(V)<\infty . \\
\text { The velocity function } a: V \rightarrow \mathbb{R}^{N} \text { lies in } \mathrm{W}^{1, \infty}(V) . \\
\text { There exist two constants } C, \gamma>0 \text { such that } \\
\qquad \mu(\{v \in V,|a(v) \cdot \xi| \leq h\}) \leq C h^{\gamma}, \quad \text { for all } \xi \in S^{N-1}, h>0 .
\end{array}\right.
$$

The assumption on $\mu$ will allow us to use the Averaging lemma, introduced by Golse et al. [25]. Next, we make precise the assumptions on the function $\sigma$ :

$$
\text { (H2) }\left\{\begin{array}{l}
\sigma\left(x, y, v, v^{\prime}\right) \in \mathrm{C}^{1}\left(\mathbb{R}^{N} ; \mathrm{W}^{1, \infty}\left(Y, \mathrm{C}^{0}(V \times V)\right)\right) . \\
\text { The function } y \longmapsto \sigma\left(x, y, v, v^{\prime}\right) \text { is } Y \text {-periodic. } \\
\text { There exists } \sigma_{*}, \sigma^{*} \in \mathrm{C}^{0}\left(\mathbb{R}^{N}\right) \text { such that } 0<\sigma_{*}(x) \leq \sigma\left(x, y, v, v^{\prime}\right) \leq \sigma^{*}(x) .
\end{array}\right.
$$

Note that the regularity assumption on $\sigma$ may not be optimal (see Lem. 3.2). However, since we shall obtain the limit equation in a weak sense, with $\rho$ only a measure with respect to $x$, it is clear we need some regularity with respect to the slow variable (in order to define, at least in the distributional sense, the products $\rho U$ and $\left.D \nabla_{x} \rho\right)$. Similarly, regularity with respect to the variables $y, v, v^{\prime}$ is related to the fact that we will need the continuity of the solutions of certain cell problems.

Finally, for a proof of convergence, we have to suppose some uniform bound on the initial data. In view of (3), the only immediate estimate on $f_{\varepsilon}$ is in the space $\mathrm{L}^{\infty}\left([0, \infty) ; \mathrm{L}^{1}\left(\mathbb{R}^{N} \times V\right)\right)$, provided we suppose that the initial data $f_{\varepsilon}^{0} \geq 0$ satisfies:

$$
\sup _{\varepsilon>0} \int_{\mathbb{R}^{N}} \int_{V} f_{\varepsilon}^{0}(x, v) \mathrm{d} \mu(v) \mathrm{d} x \leq C_{0}<\infty
$$

In this paper, we shall say nothing about the existence of a solution $f_{\varepsilon} \in \mathrm{C}^{0}\left([0, \infty) ; \mathrm{L}^{1}\left(\mathbb{R}^{N} \times V\right)\right)$ of $(1)$, for $\varepsilon>0$, with assumptions (H1-H3). Instead, we refer for instance to the paper of Petterson [39].

\subsection{Cell problems}

In this section, we are interested in the cell problem $\mathcal{T}(f)=g$, as well as the adjoint problem $\mathcal{T}^{\star}(\phi)=\psi$, with

$$
\mathcal{T}^{\star}(\phi)=-a(v) \cdot \nabla_{y} \phi-Q^{\star}(\phi),
$$

where the adjoint collision operator is given by

$$
Q^{\star}(\phi)(y, v)=\int_{Y} \sigma\left(x, y, v^{\prime}, v\right)\left(f\left(y, v^{\prime}\right)-f(y, v)\right) \mathrm{d} \mu\left(v^{\prime}\right)
$$

(note that constants belong to $\operatorname{Ker}\left(\mathcal{T}^{\star}\right)$ ). 
In order to state our results, we need to introduce some functional spaces: Let $\Omega \subset \mathbb{R}^{D}$, and $p, q \in \mathbb{N}$. We denote by $\mathrm{C}_{\#}^{p}\left(\Omega \times \mathbb{R}^{N} \times V\right)$ the set of $p$-times continuously differentiable functions on $\Omega \times \mathbb{R}^{N}$ which are $Y$-periodic with respect to the second variable. Similarly, $\mathrm{C}_{b, \#}^{p}\left(\Omega \times \mathbb{R}^{N} \times V\right)$ and $\mathrm{C}_{c, \#}^{p}\left(\Omega \times \mathbb{R}^{N} \times V\right)$ are the subspaces of functions having bounded derivatives up to order $p$, and those having support in $K \times \mathbb{R}^{N} \times V$ for some compact subset $K$ of $\Omega$, respectively. Finally, we denote by $L_{\#}^{p}\left(\Omega \times \mathbb{R}^{N} \times V\right)$ (respectively, $\left.W_{\#}^{1, p}\left(\Omega \times \mathbb{R}^{N} \times V\right) \ldots\right)$ the completion of $\mathrm{C}_{c, \#}^{\infty}\left(\Omega \times \mathbb{R}^{N} \times V\right)$ under the $L^{p}(\Omega \times Y \times V)$ norm (resp., $\left.W^{1, p}(\Omega \times Y \times V) \ldots\right)$.

In the first two results, we look at the operator $\mathcal{T}$ and $\mathcal{T}^{\star}$ for a fixed value of the parameter $x$ (all the estimates will be uniform with respect to $x$ ). The first claim describes the kernel of the operators $\mathcal{T}$, $\mathcal{T}^{\star}$, and the Fredholm alternative.

Proposition 3.1. Suppose (H1) and $\sigma \in \mathrm{C}^{0}\left(Y ; \mathrm{L}^{\infty}(V \times V)\right)$ such that $0<\sigma_{*} \leq \sigma \leq \sigma^{*}$. The following assertions hold:

i) there exists a unique function $F \in \mathrm{L}_{\#}^{p}(Y \times V)$, for $1 \leq p<\infty$, satisfying

$$
\mathcal{T}(F)=0, \quad \int_{Y} \int_{V} F \mathrm{~d} \mu(v) \mathrm{d} y=1, \quad F>0 .
$$

Similarly, we have $\operatorname{Ker}\left(\mathcal{T}^{\star}\right)=\operatorname{Span}\{\mathbb{1}\}$;

ii) let $1<p<\infty$ and $g \in \mathrm{L}_{\#}^{p}(Y \times V)$. The equation $\mathcal{T}(f)=g$ admits a solution if and only if $\int_{Y} \int_{V} g \mathrm{~d} \mu(v) \mathrm{d} y=0$. Uniqueness is guaranteed by the condition $\int_{Y} \int_{V} g \mathrm{~d} \mu(v) \mathrm{d} y=0$. Furthermore, there exists a constant $C>0$ such that

$$
\|f\|_{\mathrm{L}_{\#}^{p}(Y \times V)} \leq C\|g\|_{\mathrm{L}_{\#}^{p}(Y \times V)} ;
$$

iii) let $1<p<\infty$ and $\psi \in \mathrm{L}_{\#}^{p}(Y \times V)$. The equation $\mathcal{T}^{\star}(\phi)=\psi$ admits a solution if and only if $\int_{Y} \int_{V} \psi F \mathrm{~d} \mu(v) \mathrm{d} y=0$. Uniqueness is garanteed by the condition $\int_{Y} \int_{V} \phi \mathrm{d} \mu(v) \mathrm{d} y=0$. Furthermore, there exists a constant $C>0$ such that

$$
\|\phi\|_{\mathrm{L}_{\#}^{p}(Y \times V)} \leq C\|\psi\|_{\mathrm{L}_{\#}^{p}(Y \times V)} .
$$

Quite similar statements can be found for instance in Bal [5]. For the sake of completeness, we will give some details of the proof in the Appendix. Next, we show how the regularity of the coefficients gives regularity of the solutions of the cell problems.

Lemma 3.2. Suppose that $\sigma\left(y, v, v^{\prime}\right)$ and $\partial_{y} \sigma\left(y, v, v^{\prime}\right)$ lie in $\mathrm{C}^{0}\left(V_{v} ; L^{\infty}\left(Y \times V_{v^{\prime}}\right)\right) \cap \mathrm{C}^{0}\left(V_{v^{\prime}} ; L^{\infty}\left(Y \times V_{v}\right)\right)$. Then, for $g \in \mathrm{C}^{0}\left(V, W_{\#}^{1, p}(Y)\right), 1<p<\infty$, the solution of $\mathcal{T}(f)=g$ lies in $\mathrm{C}^{0}\left(V, W_{\#}^{1, p}(Y)\right)$. A similar conclusion holds for the adjoint equation $\mathcal{T}^{\star}(\phi)=\psi$.

Note that, using the Sobolev embedding $W^{1, p}(Y) \subset C^{0}(Y)$, for $p>N$, we deduce $f \in \mathrm{C}^{0}(V \times Y)$.

Finally, we take into account the dependence with respect to the parameter $x$.

Lemma 3.3. If $\sigma \in \mathrm{C}^{k}\left(\mathbb{R}^{N} ; \mathrm{L}_{\#}^{\infty}(Y \times V)\right)$ and $g \in \mathrm{C}^{k}\left(\mathbb{R}^{N} ; \mathrm{L}_{\#}^{p}(Y \times V)\right)$, then the solution of $\mathcal{T}(f)=g$ lies in $\mathrm{C}^{k}\left(\mathbb{R}^{N} ; \mathrm{L}_{\#}^{p}(Y \times V)\right)$. A similar conclusion holds for the adjoint equation $\mathcal{T}^{\star}(\phi)=\psi$.

In particular, with assumptions (H1, H2), combining together all the pieces, we are led to the following conclusion, which will be useful in our proof.

Corollary 3.4. Suppose (H1, H2) hold.

i) Then, $F$ and $\nabla_{x} F$ are continuous functions of their arguments.

ii) For any $g \in \mathrm{C}^{1}\left(\mathbb{R}^{N} ; \mathrm{C}^{0}\left(V, \mathrm{~W}_{\#}^{1, p}(Y)\right)\right), p>N$, satisfying the compatibility condition in Proposition 3.1, the solution of $\mathcal{T}(f)=g$ is a continuous function of its arguments as well as its first derivative with respect to $x$. A similar conclusion holds for the adjoint equation $\mathcal{T}^{\star}(\phi)=\psi$. 


\subsection{Effective coefficients}

We shall obtain in the proof the effective coefficients $U$ and $D$ by means of dual formulae. Indeed, let $\chi^{\star}(x, y, v)$ be the (vector-valued) solution of the adjoint cell equation

$$
\mathcal{T}^{\star}\left(\chi^{\star}\right)=-a(v), \quad \int_{Y} \int_{V} \chi^{\star} \mathrm{d} \mu(v) \mathrm{d} y=0 .
$$

(Of course, we assume the null flux condition (6) holds.) By using the results of Section 3.2, $\chi^{\star}$ is well-defined and its components belong to $C^{0}\left(\mathbb{R}^{N} \times Y \times V\right)$, and the first derivatives with respect to $x$ are also continuous functions. Then, we have

$$
\begin{aligned}
D(x) & =-\int_{Y} \int_{V} a(v) \otimes \chi \mathrm{d} \mu(v) \mathrm{d} y=\int_{Y} \int_{V} \mathcal{T}^{\star}\left(\chi^{\star}\right) \otimes \chi \mathrm{d} \mu(v) \mathrm{d} y \\
& =\int_{Y} \int_{V} \chi^{\star} \otimes \mathcal{T}(\chi) \mathrm{d} \mu(v) \mathrm{d} y=-\int_{Y} \int_{V} \chi^{\star} \otimes a(v) F \mathrm{~d} \mu(v) \mathrm{d} y
\end{aligned}
$$

Similarly, for the drift term, we have

$$
\begin{aligned}
U(x) & =\int_{Y} \int_{V} a(v) \lambda \mathrm{d} \mu(v) \mathrm{d} y=-\int_{Y} \int_{V} \mathcal{T}^{\star}\left(\chi^{\star}\right) \lambda \mathrm{d} \mu(v) \mathrm{d} y \\
& =-\int_{Y} \int_{V} \chi^{\star} \mathcal{T}(\lambda) \mathrm{d} \mu(v) \mathrm{d} y=\int_{Y} \int_{V} \chi^{\star} a(v) \cdot \nabla_{x} F \mathrm{~d} \mu(v) \mathrm{d} y .
\end{aligned}
$$

Positivity of the matrix $D$ relies on the following dissipative property of the operator $\mathcal{T}$ (note that this kind of property is reminiscient of general dissipative properties of Markov processes as explained by Collet [15] (see also $[32]))$.

Lemma 3.5. For all $g \in \mathcal{D}(\mathcal{T})$ we have

$$
\int_{Y} \int_{V} \mathcal{T}(g) \frac{g}{F} \mathrm{~d} \mu(v) \mathrm{d} y=\frac{1}{2} \int_{Y} \int_{V} \int_{V} \sigma\left(y, v, v^{\prime}\right) F\left(y, v^{\prime}\right)\left|\frac{g}{F}\left(y, v^{\prime}\right)-\frac{g}{F}(y, v)\right|^{2} \mathrm{~d} \mu\left(v^{\prime}\right) \mathrm{d} \mu(v) \mathrm{d} y
$$

Similarly, for $\phi \in \mathcal{D}\left(\mathcal{T}^{\star}\right)$ we have

$$
\int_{Y} \int_{V} \mathcal{T}^{\star}(\phi) \phi F \mathrm{~d} \mu(v) \mathrm{d} y=\frac{1}{2} \int_{Y} \int_{V} \int_{V} \sigma\left(y, v^{\prime}, v\right) F(y, v)\left|\phi\left(y, v^{\prime}\right)-\phi(y, v)\right|^{2} \mathrm{~d} \mu\left(v^{\prime}\right) \mathrm{d} \mu(v) \mathrm{d} y .
$$

Proof. We shall only detail the computations for the adjoint operator. Setting $\phi=g / F$ and noticing that $\int_{Y} \int_{V} \mathcal{T}^{\star}(\phi) \phi F \mathrm{~d} \mu(v) \mathrm{d} y=\int_{Y} \int_{V} \mathcal{T}(g) g / F \mathrm{~d} \mu(v) \mathrm{d} y$, the result for $\mathcal{T}$ follows.

The keypoint is the (pointwise!) equality

$$
Q^{\star}(\phi) \phi F=-\frac{1}{2} \int_{V} \sigma\left(v^{\prime}, v\right) F(v)\left|\phi\left(v^{\prime}\right)-\phi(v)\right|^{2} \mathrm{~d} \mu\left(v^{\prime}\right)+Q^{\star}\left(\phi^{2}\right) \frac{F}{2}
$$


(this relation actually holds for any $\phi$ and $F$ and does not use the equation satisfied by $F$ ), which yields:

$$
\begin{aligned}
\int_{Y} \int_{V} \mathcal{T}^{\star}(\phi) \phi F \mathrm{~d} \mu(v) \mathrm{d} y= & -\int_{Y} \int_{V} a(v) \cdot \nabla_{y}\left(\frac{\phi^{2}}{2}\right) F \mathrm{~d} \mu(v) \mathrm{d} y-\int_{Y} \int_{V} Q^{\star}(\phi) \phi F \mathrm{~d} \mu(v) \mathrm{d} y \\
= & +\int_{Y} \int_{V} \frac{\phi^{2}}{2} a(v) \cdot \nabla_{y} F \mathrm{~d} \mu(v) \mathrm{d} y-\int_{Y} \int_{V} \frac{\phi^{2}}{2} Q(F) \mathrm{d} \mu(v) \mathrm{d} y \\
& +\frac{1}{2} \int_{Y} \int_{V} \int_{V} \sigma\left(v^{\prime}, v\right) F(v)\left|\phi\left(v^{\prime}\right)-\phi(v)\right|^{2} \mathrm{~d} \mu\left(v^{\prime}\right) \mathrm{d} \mu(v) \mathrm{d} y \\
= & \frac{1}{2} \int_{Y} \int_{V} \int_{V} \sigma\left(v^{\prime}, v\right) F(v)\left|\phi\left(v^{\prime}\right)-\phi(v)\right|^{2} \mathrm{~d} \mu\left(v^{\prime}\right) \mathrm{d} \mu(v) \mathrm{d} y
\end{aligned}
$$

since $a(v) \cdot \nabla_{y} F-Q(F)=0$.

It remains to justify (13). First of all, we compute

$$
\begin{aligned}
Q^{\star}(\phi) \phi F(v)= & \int_{V} \sigma\left(v^{\prime}, v\right)\left(\phi\left(v^{\prime}\right)-\phi(v)\right) \phi(v) F(v) \mathrm{d} \mu\left(v^{\prime}\right)=-\int_{V} \sigma\left(v^{\prime}, v\right) F(v)\left(\phi\left(v^{\prime}\right)-\phi(v)\right)^{2} \mathrm{~d} \mu\left(v^{\prime}\right) \\
& +\int_{V} \sigma\left(v^{\prime}, v\right)\left(\phi\left(v^{\prime}\right)-\phi(v)\right) \phi\left(v^{\prime}\right) F(v) \mathrm{d} \mu\left(v^{\prime}\right) .
\end{aligned}
$$

We rewrite the last integral as follows:

$$
\begin{array}{r}
\int_{V} \sigma\left(v^{\prime}, v\right) \phi\left(v^{\prime}\right)^{2} \mathrm{~d} \mu\left(v^{\prime}\right) F(v)-\int_{V} \sigma\left(v^{\prime}, v\right) \phi\left(v^{\prime}\right) \mathrm{d} \mu\left(v^{\prime}\right) \phi F(v) \\
=Q^{\star}\left(\phi^{2}\right) F(v)+\int_{V} \sigma\left(v^{\prime}, v\right) \mathrm{d} \mu\left(v^{\prime}\right) \phi^{2}(v) F(v)-Q^{\star}(\phi) \phi F(v) \\
\quad-\int_{V} \sigma\left(v^{\prime}, v\right) \mathrm{d} \mu\left(v^{\prime}\right) \phi^{2}(v) F(v)=Q^{\star}\left(\phi^{2}\right) F(v)-Q^{\star}(\phi) \phi F(v),
\end{array}
$$

which leads to (13). Note that, similarly, we can prove

$$
2 Q(g) g / F=Q\left(g^{2} / F\right)+Q(F)(g / F)^{2}-\int_{Y} \int_{V} \int_{V} \sigma\left(v, v^{\prime}\right) F\left(v^{\prime}\right)\left|\frac{g}{F}\left(v^{\prime}\right)-\frac{g}{F}(v)\right|^{2} \mathrm{~d} \mu\left(v^{\prime}\right) \mathrm{d} \mu(v) \mathrm{d} y
$$

As a consequence, we can show that we are effectively led to a diffusion process in the limit $\varepsilon \rightarrow 0$, since the symmetric part of $D$ is positive definite.

Proposition 3.6. The effective drift coefficient $U$ is a continuous function of $x$; the components of $D$ are $\mathrm{C}^{1}$ functions of $x$. For any $x \in \mathbb{R}^{N}, \xi \in \mathbb{R}^{N} \backslash\{0\}$, we have $D(x) \xi \cdot \xi>0$.

Proof. The regularity of the coefficients follows immediately from the regularity of $\chi^{\star}$ and $F$. Positivity of $D \xi \cdot \xi$ follows from the duality formula (11) combined with Lemma 3.5. Indeed, since $\mathcal{T}^{\star}\left(\chi^{\star}\right)=-a(v)$, we obtain

$$
\begin{aligned}
D \xi \cdot \xi & =-\int_{Y} \int_{Y} \chi^{\star} \cdot \xi a(v) \cdot \xi F \mathrm{~d} \mu(v) \mathrm{d} y=\int_{Y} \int_{V} \chi^{\star} \cdot \xi \mathcal{T}^{\star}\left(\chi^{\star} \cdot \xi\right) F \mathrm{~d} \mu(v) \mathrm{d} y \\
& =\frac{1}{2} \int_{Y} \int_{V} \int_{V} \sigma\left(v^{\prime}, v\right) F(v)\left|\chi^{\star}\left(v^{\prime}\right) \cdot \xi-\chi^{\star}(v) \cdot \xi\right|^{2} \mathrm{~d} \mu\left(v^{\prime}\right) \mathrm{d} \mu(v) \mathrm{d} y \geq 0 .
\end{aligned}
$$

Furthermore, since $\sigma F>0$, this quantity vanishes when $\chi^{\star} \cdot \xi$ does not depend on $v$. In this case, we have $Q^{\star}\left(\chi^{\star} \cdot \xi\right)=0$ and the cell equation reduces to $\mathcal{T}^{\star}\left(\chi^{\star} \cdot \xi\right)=-a(v) \cdot \nabla_{y}\left(\chi^{\star} \cdot \xi\right)=-a(v) \cdot \xi$, which does not depend on $y$. The periodicity implies that, for any $v, a(v) \cdot \xi=0$; thus $\xi=0$. 


\subsection{Functional preliminaries}

In the present problem there is no "immediate" a priori $\mathrm{L}^{2}$-estimates, and the natural estimate is in $\mathrm{L}^{1}$ (see (3)). For this reason, we need to introduce some definitions and basic results about families of parametrized measures. In what follows, we shall denote by $\mathcal{M}^{1}(\Omega)$ the set of Radon measures on $\Omega$.

Definition 3.7. Let $I$ be an interval of $\mathbb{R}$. A family $\{\rho(t) ; t \in I\}$ of Radon measures on $\mathbb{R}^{N}$ is said to be vaguely continuous if and only if for any $\varphi \in C_{c}^{0}\left(\mathbb{R}^{N}\right)$,

$$
t \longmapsto \int_{\mathbb{R}^{N}} \varphi(x) \rho(t, \mathrm{~d} x) \text { is a continuous function on } I \text {. }
$$

Definition 3.8. A sequence $\left\{\rho_{n}(t) ; t \in I, n \in \mathbb{N}\right\}$ of parametrized measures on $\mathbb{R}^{N}$ is said to be equibounded and vaguely equicontinuous on $I$ if and only if

i) there exists $M>0$ such that

$$
\sup _{t \in I, n \in \mathbb{N}}\left|\rho_{n}(t)\right|\left(\mathbb{R}^{N}\right) \leq M
$$

ii) for any $\varphi \in C_{c}^{0}\left(\mathbb{R}^{N}\right)$, the sequence of functions $\left(t \longmapsto \int_{\mathbb{R}^{N}} \varphi(x) \rho_{n}(t, \mathrm{~d} x)\right)_{n \in \mathbb{N}}$ is equicontinuous on $I$.

Classical methods of analysis lead to the following compactness property:

Proposition 3.9. Let $I$ be an interval of $\mathbb{R}$. Let $\left(\rho_{n}(t)\right)_{n \in \mathbb{N}}$ be a sequence of Radon measures on $\mathbb{R}^{N}$, equibounded and vaguely equicontinuous on $I$. Then, there exists a measure $\rho(t)$, which is vaguely continuous on $I$, and a subsequence $\left(\rho_{n_{k}}(t)\right)_{k \in \mathbb{N}}$ such that for any $\varphi \in C_{c}^{0}\left(I \times \mathbb{R}^{N}\right)$,

$$
\int_{\mathbb{R}^{N}} \varphi(t, x) \rho_{n_{k}}(t, \mathrm{~d} x) \underset{k \rightarrow \infty}{\longrightarrow} \int_{\mathbb{R}^{N}} \varphi(t, x) \rho(t, \mathrm{~d} x),
$$

uniformly with respect to $t \in I$. We say that the sequence $\left(\rho_{n_{k}}(t)\right)_{k \in \mathbb{N}}$ converges vaguely to $\rho(t)$ locally, uniformly on $I$.

Next, we need a measure-valued version of the existence of double-scale limit, as introduced by N'Guetseng [38] and Allaire [1].

Proposition 3.10. Let $\left(\varepsilon_{n}\right)_{n \in \mathbb{N}}$ be a sequence of positive numbers converging to 0 . Let $\left(\mu_{n}(t)\right)_{n \in \mathbb{N}}$ be a sequence of measures on $[0, \infty) \times \mathbb{R}^{N} \times V$ which satisfies, for any bounded interval $I \subset \mathbb{R}$,

$$
\sup _{n \in \mathbb{N}}\left|\mu_{n}\right|\left(I \times \mathbb{R}^{N} \times V\right) \leq C(I)<\infty
$$

Then, there exists a subsequence $\left(\mu_{n_{k}}(t)\right)_{k \in \mathbb{N}}$ and a measure $M$ on $[0, \infty) \times \mathbb{R}^{N} \times Y \times V$ such that, for any $\varphi \in C_{c, \#}^{0}\left(I \times \mathbb{R}^{N} \times \mathbb{R}^{N} \times V\right)$, we have

$$
\int_{0}^{\infty} \int_{\mathbb{R}^{N}} \int_{V} \varphi\left(t, x, x / \varepsilon_{n_{k}}, v\right) \mu_{n_{k}}(\mathrm{~d} v, \mathrm{~d} x, \mathrm{~d} t) \underset{k \rightarrow \infty}{\longrightarrow} \int_{0}^{\infty} \int_{\mathbb{R}^{N}} \int_{Y} \int_{V} \varphi(t, x, y, v) M(\mathrm{~d} v, \mathrm{~d} y, \mathrm{~d} x, \mathrm{~d} t)
$$

We say that $M$ is the double-scale limit of the sequence $\left(\mu_{n_{k}}(t)\right)_{k \in \mathbb{N}}$.

This proposition is just a consequence of the Banach-Alaoglu theorem (see e.g. [10]) applied to the sequence of measures $M_{n}$ defined by

$$
\int_{[0,+\infty) \times \mathbb{R}^{N} \times Y \times V}^{\varphi(t, x, y, v)} M_{n}(\mathrm{~d} v, \mathrm{~d} y, \mathrm{~d} x, \mathrm{~d} t)=\int_{[0,+\infty) \times \mathbb{R}^{N} \times V} \varphi\left(t, x, x / \varepsilon_{n}, v\right) \mu_{n}(\mathrm{~d} v, \mathrm{~d} x, \mathrm{~d} t) .
$$


The double-scale limit captures the periodic oscillations of $\mu_{n}$ which have frequency $1 / \varepsilon_{n}$ with respect to the variable $x$. Actually, if $\mu_{n}$ is obtained from a sequence of functions bounded in $\mathrm{L}^{\infty}\left([0, \infty) ; \mathrm{L}^{1}\left(\mathbb{R}^{N} \times V\right)\right)$, then the double-scale limit $M$ has a slightly better regularity with respect to time: it belongs to the set $\mathrm{L}_{w}^{\infty}\left([0, \infty) ; \mathcal{M}^{1}\left(\mathbb{R}^{N} \times Y \times V\right)\right.$ of weakly-* measurable functions $M: t \in(0, T) \longrightarrow M(t) \in \mathcal{M}^{1}\left(\mathbb{R}^{N} \times Y \times V\right)$ satisfying

$$
\sup _{0 \leq t \leq T}\|M(t)\|_{\mathcal{M}^{1}}<\infty
$$

This set is identified with the dual of $L^{1}\left(0, T ; C_{0}^{0}\left(\mathbb{R}^{N} \times Y \times V\right)\right)$. We refer the reader to [20] (Chap. 8, 18) for details of these aspects. As usual, the vague limit $\mu$ of $\mu_{n_{k}}$ in $\mathcal{M}^{1}\left(I \times \mathbb{R}^{N} \times V\right)$ is given by the marginal of the double scale limit $\mu(\mathrm{d} v, \mathrm{~d} x, \mathrm{~d} t)=\int_{Y} M(\mathrm{~d} v, \mathrm{~d} y, \mathrm{~d} x, \mathrm{~d} t)$.

\subsection{Convergence result}

We are now in position to give the statement of our main result.

Theorem 3.11. Suppose (H1-H3) hold. Suppose that the velocity field $a: V \rightarrow \mathbb{R}^{N}$ is such that the normalized solution of $\mathcal{T}(F)=0$ satisfies

$$
\int_{Y} \int_{V} a(v) F(x, y, v) \mathrm{d} \mu(v) \mathrm{d} y=0 .
$$

Moreover, suppose that $\rho_{\varepsilon}^{0}=\int_{V} f_{\varepsilon}^{0} \mathrm{~d} \mu(v)$ converges vaguely to $\rho^{0}$ in $\mathcal{M}^{1}\left(\mathbb{R}^{N}\right)$. Then, up to a subsequence, $\rho_{\varepsilon}(t, x)=\int_{V} f_{\varepsilon} \mathrm{d} \mu(v)$ converges to $\rho$ vaguely, uniformly on any time interval $[0, T]$. The limit satisfies the drift-diffusion equation

$$
\partial_{t} \rho-\operatorname{div}_{x}\left(D \nabla_{x} \rho\right)+\operatorname{div}_{x}(U \rho)=0
$$

in $\mathcal{D}^{\prime}\left([0, \infty) \times \mathbb{R}^{N}\right)$ with Cauchy data $\rho^{0}$. The effective coefficients are defined by

$$
\left\{\begin{array}{l}
D(x)=-\int_{Y} \int_{V} \chi^{\star}(x, y, v) \otimes a(v) F(x, y, v) \mathrm{d} \mu(v) \mathrm{d} y, \\
U(x)=\int_{Y} \int_{V} \chi^{\star}(x, y, v) a(v) \cdot \nabla_{x} F(x, y, v) \mathrm{d} \mu(v) \mathrm{d} y,
\end{array}\right.
$$

with $\chi^{\star}$ solution of the cell problem $\mathcal{T}^{\star}\left(\chi^{\star}\right)=-a(v)$.

The next section is devoted to the proof of this theorem.

\section{The VANishing FLUX CASE}

The proof of Theorem 3.11 relies on the use of oscillating test functions, in the spirit of the works by Tartar [42] or Evans [21,22]. The method has been used by Goudon and Poupaud [30] when dealing with homogenization of general advection-diffusion equations. Let us recall that the only immediate estimate we have deals with the $\mathrm{L}^{1}$-norm of $f_{\varepsilon}(3)$.

Step 1: Duality Relations and Compactness of $\rho_{\varepsilon}$.

Let us multiply the equation (1) by a test function $\varphi\left(t, x, \frac{x}{\varepsilon}, v\right)$ with $\varphi \in C_{c, \#}^{1}\left([0, \infty) \times \mathbb{R}^{N} \times \mathbb{R}^{N} \times V\right)$. We get

$$
\begin{aligned}
\frac{\mathrm{d}}{\mathrm{d} t} \int_{\mathbb{R}^{N}} \int_{V} f_{\varepsilon}(t, x) \varphi(t, x, x / \varepsilon, v) \mathrm{d} \mu(v) \mathrm{d} x & = \\
& \int_{\mathbb{R}^{N}} \int_{V} f_{\varepsilon}(t, x)\left(\partial_{t} \varphi+\frac{1}{\varepsilon} a(v) \cdot \nabla_{x} \varphi+\frac{1}{\varepsilon^{2}} a(v) \cdot \nabla_{y} \varphi+\frac{1}{\varepsilon^{2}} Q^{\star}(\varphi)\right)(t, x, x / \varepsilon, v) \mathrm{d} \mu(v) \mathrm{d} x
\end{aligned}
$$


- As a first consequence, for any such test function $\varphi$, we have

$$
\lim _{\varepsilon \rightarrow 0} \int_{0}^{\infty} \int_{\mathbb{R}^{N}} \int_{V} f_{\varepsilon}(t, x) \mathcal{T}^{\star}(\varphi)(t, x, x / \varepsilon, v) \mathrm{d} \mu(v) \mathrm{d} x \mathrm{~d} t=0 .
$$

- Then, choosing a test function $\varphi(t, x, x / \varepsilon, v)=\phi(t, x)+\varepsilon \psi(t, x, x / \varepsilon, v)$, we get rid of the $\varepsilon^{-2}$ terms in (14) (since $\phi(t, x)$ lies in the kernel of $\mathcal{T}^{\star}$ ), and we get:

$$
\lim _{\varepsilon \rightarrow 0} \int_{0}^{\infty} \int_{\mathbb{R}^{N}} \int_{V} f_{\varepsilon}(t, x)\left(a(v) \cdot \nabla_{x} \phi(t, x)-\mathcal{T}^{\star}(\psi)(t, x, x / \varepsilon, v)\right) \mathrm{d} \mu(v) \mathrm{d} x \mathrm{~d} t=0,
$$

which yields, in view of (15),

$$
\lim _{\varepsilon \rightarrow 0} \int_{0}^{\infty} \int_{\mathbb{R}^{N}} \int_{V} f_{\varepsilon}(t, x) a(v) \cdot \nabla_{x} \phi(t, x) \mathrm{d} \mu(v) \mathrm{d} x \mathrm{~d} t=0 .
$$

It is worth rewriting this relation as a limit equation for the macroscopic current $J_{\varepsilon}(t, x)=\int_{V} \frac{a(v)}{\varepsilon} f_{\varepsilon} \mathrm{d} \mu(v)$. Here, we have no bound on $J_{\varepsilon}$, but (16) says that

$$
\lim _{\varepsilon \rightarrow 0} \int_{0}^{\infty} \int_{\mathbb{R}^{N}} \varepsilon J_{\varepsilon} \cdot \nabla_{x} \psi(t, x) \mathrm{d} x \mathrm{~d} t=0
$$

- We now choose the $\varepsilon$-correction, $\psi(t, x, y, v)$, to be solution to the following adjoint cell equation:

$$
\mathcal{T}^{\star}(\psi)=a(v) \cdot \nabla_{x} \phi(t, x) .
$$

We therefore have $\psi(t, x, y, v)=-\chi^{\star}(x, y, v) \cdot \nabla_{x} \phi(t, x)$, with $\chi^{\star}$ the vector-valued function defined by

$$
\mathcal{T}^{\star}\left(\chi^{\star}\right)=-a(v), \quad \int_{Y} \int_{V} \chi^{\star} \mathrm{d} \mu(v) \mathrm{d} y=0 .
$$

By Corollary 3.4 , since $\phi \in \mathrm{C}_{c}^{2}\left([0, \infty) \times \mathbb{R}^{N}\right)$, the functions $\psi$ and $\nabla_{x} \psi$ belong to $\mathrm{C}_{c, \#}^{0}\left([0, \infty) \times \mathbb{R}^{N} \times \mathbb{R}^{N} \times V\right)$. With this particular choice of test function, the singular terms in (14) vanish and we get

$$
\begin{aligned}
\frac{\mathrm{d}}{\mathrm{d} t} \int_{\mathbb{R}^{N}} \int_{V} f_{\varepsilon}(t, x)(\phi(t, x)+\varepsilon \psi(t, x, x / \varepsilon, v)) \mathrm{d} \mu(v) \mathrm{d} x= & \int_{\mathbb{R}^{N}} \int_{V} f_{\varepsilon}(t, x)\left(\partial_{t} \phi(t, x)+\varepsilon \partial_{t} \psi(t, x, x / \varepsilon, v)\right) \mathrm{d} \mu(v) \mathrm{d} x \\
& +\int_{\mathbb{R}^{N}} \int_{V} f_{\varepsilon}(t, x) a(v) \cdot\left(\nabla_{x} \psi\right)(t, x, x / \varepsilon, v) \mathrm{d} \mu(v) \mathrm{d} x
\end{aligned}
$$

The last integral reads ${ }^{4}$

$$
-\int_{\mathbb{R}^{N}} \int_{V} f_{\varepsilon}(t, x)\left(a(v) \otimes \chi^{\star}(x, x / \varepsilon, v): D_{x}^{2} \phi(t, x)+\left(D_{x} \chi^{\star}\right)^{T}(x, x / \varepsilon, v) a(v) \cdot \nabla_{x} \phi(t, x)\right) \mathrm{d} \mu(v) \mathrm{d} x .
$$

In (17), the right hand side is bounded, uniformly with respect to $\varepsilon$. We deduce that, for any given function $\phi \in C_{c}^{2}\left([0, \infty) \times \mathbb{R}^{N}\right)$, the family

$$
t \longmapsto \int_{\mathbb{R}^{N}} \int_{V} f_{\varepsilon}(t, x)(\phi(t, x)+\varepsilon \psi(t, x, x / \varepsilon, v)) \mathrm{d} \mu(v) \mathrm{d} x
$$

\footnotetext{
${ }^{4}$ For a vector valued function $g: \mathbb{R}^{N} \rightarrow \mathbb{R}^{N}, D_{x} g$ stands for the Jacobian matrix $\partial_{j} g_{i}$. For two $N \times N$ matrices $A, B$, we denote $A: B=\operatorname{tr}\left(A B^{T}\right)=\sum_{i j=1}^{N} A_{i j} B_{i j}$.
} 
is equicontinuous. Since the functions

$$
t \longmapsto \int_{\mathbb{R}^{N}} \int_{V} f_{\varepsilon} \phi(t, x) \mathrm{d} \mu(v) \mathrm{d} x=\int_{\mathbb{R}^{N}} \rho_{\varepsilon} \phi(t, x) \mathrm{d} x
$$

are close, up to $\mathcal{O}(\varepsilon)$, to the previous ones, they also form an equicontinuous family of functions. By density of $C_{c}^{2}\left(\mathbb{R}^{N}\right)$ in $C_{c}^{0}\left(\mathbb{R}^{N}\right)$, we conclude that the family of nonnegative measures $\left(\rho^{\varepsilon}(t)\right)_{\varepsilon>0}$ is vaguely equicontinuous on $[0, \infty)$.

\section{Step 2: Passage to the limit.}

We can therefore suppose that $\rho_{\varepsilon}$ converges to $\rho$ in the sense of Proposition 3.9, and that the nonnegative measure $f_{\varepsilon}(t, x, v) \mathrm{d} \mu(v) \mathrm{d} x \mathrm{~d} t$ converges to the double scale limit $\mathcal{F}$, in the sense of Proposition 3.10. Clearly, these limits are related by

$$
\rho(t, \mathrm{~d} x) \mathrm{d} t=\int_{Y} \int_{V} \mathcal{F}(\mathrm{d} v, \mathrm{~d} y, \mathrm{~d} x, \mathrm{~d} t),
$$

i.e., $\rho$ is the marginal with respect to $t, x$ of $\mathcal{F}$ (note, however, that equicontinuity with respect to time is far from obvious for the sequence of microscopic quantities $f_{\varepsilon}$ ). It remains to identify these limits.

Lemma 4.1. The double scale limit $\mathcal{F}$ is given by

$$
\mathcal{F}(\mathrm{d} v, \mathrm{~d} y, \mathrm{~d} x, \mathrm{~d} t)=F(x, y, v) \mathrm{d} \mu(v) \mathrm{d} y \rho(t, \mathrm{~d} x) \mathrm{d} t .
$$

Remark 4.2. Roughly speaking, this result says that $f_{\varepsilon}(t, x, v)$ behaves like the product $\rho(t, x) F(x, x / \varepsilon, v)$, with $\rho$ a solution of a drift-diffusion equation. In particular, oscillations of the equilibrium function obstruct strong convergence, contrary to the situation dealt with in $[27,29]$.

Let us postpone the proof of Lemma 4.1, and proceed to the limit $\varepsilon \rightarrow 0$ in (17). In the sense of distributions on $[0, \infty) \times \mathbb{R}^{N}$, we get:

$$
\begin{aligned}
& \left\langle\partial_{t} \rho, \phi\right\rangle=-\int_{\mathbb{R}^{N}} \phi(0, x) \rho^{0}(\mathrm{~d} x)-\int_{0}^{\infty} \int_{\mathbb{R}^{N}} \partial_{t} \phi(t, x) \rho(t \mathrm{~d} x) \mathrm{d} t \\
& =-\int_{0}^{\infty} \int_{\mathbb{R}^{N}} \int_{Y \times V}\left(a(v) \otimes \chi^{\star}: D_{x}^{2} \phi+\left(D_{x} \chi^{\star}\right)^{T} a(v) \cdot \nabla_{x} \phi\right) \mathcal{F}(\mathrm{d} v, \mathrm{~d} y, \mathrm{~d} x, \mathrm{~d} t) \\
& =-\int_{0}^{\infty} \int_{\mathbb{R}^{N}}\left[\int_{Y \times V} a(v) F \otimes \chi^{\star} \mathrm{d} \mu(v) \mathrm{d} y: D_{x}^{2} \phi\right. \\
& \left.+\int_{Y \times V}\left(D_{x} \chi^{\star}\right)^{T} a(v) F \mathrm{~d} \mu(v) \mathrm{d} y \cdot \nabla_{x} \phi\right] \rho(t, \mathrm{~d} x) \mathrm{d} t \\
& =-\int_{0}^{\infty} \int_{\mathbb{R}^{N}}^{\infty V}\left[\nabla_{x} \cdot\left(\int_{Y \times V} a(v) F \otimes \chi^{\star} \mathrm{d} \mu(v) \mathrm{d} y \nabla_{x} \phi\right)\right. \\
& \left.-\int_{Y \times V} \chi^{\star} a(v) \cdot \nabla_{x} F \mathrm{~d} \mu(v) \mathrm{d} y \cdot \nabla_{x} \phi\right] \rho(t, \mathrm{~d} x) \mathrm{d} t \\
& =\int_{0}^{\infty} \int_{\mathbb{R}^{N}}^{\infty}\left(\nabla_{x} \cdot\left(D^{T} \nabla_{x} \phi\right)+U \nabla_{x} \phi\right) \rho(t, \mathrm{~d} x) \mathrm{d} t \\
& =\left\langle\nabla_{x} \cdot\left(D \nabla_{x} \rho-U \rho\right), \phi\right\rangle \text {, }
\end{aligned}
$$

which is nothing but the drift-diffusion equation for $\rho$. Note that the effective coefficients are defined by the duality formulae (11) and (12).

Proof of Lemma 4.1. In order to complete the proof, it remains to establish Lemma 4.1. Coming back to (15), we have:

$$
\int_{0}^{\infty} \int_{\mathbb{R}^{N}} \int_{Y} \int_{V} \mathcal{T}^{\star}(\varphi) \mathcal{F}(\mathrm{d} v, \mathrm{~d} y, \mathrm{~d} x, \mathrm{~d} t)=0 .
$$


Formally, it means that $\mathcal{F}$ lies in $\left(\operatorname{Ran}\left(\mathcal{T}^{\star}\right)\right)^{\perp}=\operatorname{Ker}(\mathcal{T}$ ) which leads to $\mathcal{F}(t, x, y, v)=\bar{\rho}(t, x) F(x, y, v)$ (with $\bar{\rho}(t, x)=\rho(t, x)$, since the equilibrium function $F$ is normalized). However, the lack of regularity of the double scale limit, which is only a measure, does not allow to use this direct argument. Instead, let us consider a smooth function $H$ such that $\int_{Y} \int_{V} H F \mathrm{~d} v \mathrm{~d} y=0$. By Proposition 3.1, it can be written as $H=\mathcal{T}^{\star}(\varphi)$ and we get, by (15),

$$
\int_{0}^{\infty} \int_{\mathbb{R}^{N}} \int_{Y} \int_{V} H \mathcal{F}(\mathrm{d} v, \mathrm{~d} y, \mathrm{~d} x, \mathrm{~d} t)=0 .
$$

Let $\varphi$ be any test function. We split it as follows:

$$
\left\{\begin{array}{l}
\varphi(t, x, y, v)=c_{\varphi}(t, x) F(x, y, v)+\left(\varphi(t, x, y, v)-c_{\varphi}(t, x) F(x, y, v)\right) \\
c_{\varphi}(t, x)=\int_{Y} \int_{V} \varphi(t, x, y, v) F(x, y, v) \mathrm{d} \mu(v) \mathrm{d} y\left(\int_{Y} \int_{V} F^{2}(x, y, v) \mathrm{d} \mu(v) \mathrm{d} y\right)^{-1}
\end{array}\right.
$$

By definition $\int_{Y} \int_{V}\left(\varphi-c_{\varphi} F\right) F \mathrm{~d} \mu(v) \mathrm{d} y=0$, and we have

$$
\begin{aligned}
\int_{0}^{\infty} \int_{\mathbb{R}^{N}} \int_{Y} \int_{V} \varphi(t, x, y, v) \mathcal{F}(\mathrm{d} v, \mathrm{~d} y, \mathrm{~d} x, \mathrm{~d} t) & =\int_{0}^{\infty} \int_{\mathbb{R}^{N}} \int_{Y} \int_{V} c_{\varphi}(t, x) F(x, y, v) \mathcal{F}(\mathrm{d} v, \mathrm{~d} y, \mathrm{~d} x, \mathrm{~d} t) \\
& =\int_{0}^{\infty} \int_{\mathbb{R}^{N}} \int_{Y} \int_{V} \varphi(t, x, z, w) F(x, z, w) \mathrm{d} \mu(w) \mathrm{d} z \bar{\rho}(\mathrm{d} x, \mathrm{~d} t)
\end{aligned}
$$

where the (nonnegative) measure $\bar{\rho} \in \mathcal{M}^{1}\left(I \times \mathbb{R}^{N}\right)$ is defined by

$$
\bar{\rho}(\mathrm{d} x, \mathrm{~d} t)=\left(\int_{Y} \int_{V} F^{2}(x, y, v) \mathrm{d} \mu(v) \mathrm{d} y\right)^{-1} \int_{Y} \int_{V} F(x, y, v) \mathcal{F}(\mathrm{d} v, \mathrm{~d} y, \mathrm{~d} x, \mathrm{~d} t) .
$$

It follows that $F(\mathrm{~d} v, \mathrm{~d} y, \mathrm{~d} x, \mathrm{~d} t)=F(x, y, v) \mathrm{d} \mu(v) \mathrm{d} y \bar{\rho}(\mathrm{d} x, \mathrm{~d} t)$. And when $\varphi$ only depends on $t, x$, the previous computation yields

$$
\bar{\rho}(\mathrm{d} x, \mathrm{~d} t)=\rho(t, \mathrm{~d} x) \mathrm{d} t .
$$

\section{THE NON-VANISHING FLUX CASE}

All the results we derived up to now have been obtained under the hypothesis that the flux of the equilibrium function vanishes. Nevertheless, such an hypothesis could be violated in physical situations. In this section, we shall therefore investigate the asymptotic behaviour of $f^{\varepsilon}$ when the null flux condition (6) is no longer fulfilled. Other attempts on this question can be found in [12].

\subsection{The general case}

We introduce the flux of the equilibrium function:

$$
c(x)=\int_{Y} \int_{V} a(v) F(x, y, v) \mathrm{d} \mu(v) \mathrm{d} y \neq 0 .
$$

It is then possible to adapt the proof of Theorem 3.11, and establish the following result:

Theorem 5.1. Suppose that (H1-H3) hold. Assume moreover that $\rho_{\varepsilon}^{0}=\int_{V} f_{\varepsilon}^{0} \mathrm{~d} \mu(v)$ converges vaguely to $\rho^{0}$ in $\mathcal{M}^{1}\left(\mathbb{R}^{N}\right)$. Then, up to a subsequence, $\rho_{\varepsilon}(t, x)=\int_{V} f_{\varepsilon} \mathrm{d} \mu(v)$ converges to $\rho \geq 0$ vaguely in $\mathcal{M}^{1}\left((0, T) \times \mathbb{R}^{N}\right)$; the limit satisfies

$$
\operatorname{div}_{x}(c \rho)=0 \quad \text { in } \mathcal{D}^{\prime}\left((0, \infty) \times \mathbb{R}^{N}\right) .
$$


Moreover, for any $\varphi \in \mathrm{C}_{c}^{\infty}\left([0, \infty) \times \mathbb{R}^{N}\right)$ satisfying $c \cdot \nabla_{x} \varphi=0$, we have

$$
\int_{\mathbb{R}^{N}} \varphi \rho_{\varepsilon}(t, x) \mathrm{d} x \underset{\varepsilon \rightarrow 0}{\longrightarrow} \int_{\mathbb{R}^{N}} \varphi \rho(t, \mathrm{~d} x)
$$

uniformly on any time interval $[0, T]$. Moreover, the following relation holds:

$$
\frac{\mathrm{d}}{\mathrm{d} t} \int_{\mathbb{R}^{N}} \varphi \rho(t, \mathrm{~d} x)=\int_{\mathbb{R}^{N}}\left(\partial_{t} \varphi-U \cdot \nabla_{x} \varphi+\nabla_{x} \cdot\left(D^{T} \nabla_{x} \varphi\right)\right) \rho(t, \mathrm{~d} x),
$$

with $\int_{\mathbb{R}^{N}} \varphi \rho(0, \mathrm{~d} x)=\int_{\mathbb{R}^{N}} \varphi \rho^{0}(\mathrm{~d} x)$. The effective coefficients are defined by

$$
\left\{\begin{array}{l}
D(x)=-\int_{Y} \int_{V} \chi^{\star}(x, y, v) \otimes(a(v)-c) F(x, y, v) \mathrm{d} \mu(v) \mathrm{d} y \\
U(x)=\int_{Y} \int_{V} \chi^{\star}(x, y, v)(a(v)-c) \cdot \nabla_{x} F(x, y, v) \mathrm{d} \mu(v) \mathrm{d} y
\end{array}\right.
$$

with $\chi^{\star}$ a solution of the cell problem $\mathcal{T}^{\star}\left(\chi^{\star}\right)=-(a(v)-c)$.

Proof. We follow the proof of Theorem 3.11 with some slight modifications. First, we modify (16) as follows:

$$
\begin{aligned}
\int_{0}^{\infty} \int_{\mathbb{R}^{N}} \int_{V} f_{\varepsilon}(t, x)\left((a(v)-c) \cdot \nabla_{x} \phi(t, x)-\mathcal{T}^{\star}(\psi)(t, x, x / \varepsilon, v)\right) \mathrm{d} \mu(v) \mathrm{d} x \mathrm{~d} t & \\
& \quad+\int_{0}^{\infty} \int_{\mathbb{R}^{N}} \int_{V} f_{\varepsilon} c \cdot \nabla_{x} \phi(t, x) \mathrm{d} \mu(v) \mathrm{d} x \mathrm{~d} t \underset{\varepsilon \rightarrow 0}{\longrightarrow} 0 .
\end{aligned}
$$

Then, we choose the function $\psi$ so that the first integral vanishes, i.e. $\psi(t, x, y, v)=\chi^{\star}(x, y, v) \cdot \nabla_{x} \psi$, with $\mathcal{T}^{\star}\left(\chi^{\star}\right)=-(a(v)-c)$. It leads to the constraint

$$
\lim _{\varepsilon \rightarrow 0} \int_{0}^{\infty} \int_{\mathbb{R}^{N}} \rho^{\varepsilon} c \cdot \nabla_{x} \phi \mathrm{d} x \mathrm{~d} t=0 .
$$

And finally, fixing a test function $\phi$, satisfying $c \cdot \nabla_{x} \phi=0$, we can reproduce the arguments of the proof of Theorem 3.11 .

It is very tempting to describe the orthogonality constraint in (18) by means of Lagrange multiplier. We interpret the condition $c \cdot \nabla_{x} \varphi=0$ as $\varphi \in \operatorname{Ker}(A)$ with $A$ the symmetric operator $A=\nabla_{x}\left(c \otimes c \nabla_{x} \cdot\right)$. Hence, formally, a distribution $T \in(\operatorname{Ker} A)^{\perp}$ lies in $\operatorname{Ran}(A)$ and it can be rewritten $T=\operatorname{div}_{x}(c p), p=c \cdot \nabla q$. Thus, equation (18) becomes $\partial_{t} \rho-\operatorname{div}_{x}\left(D \nabla_{x} \rho-U \rho\right)=\operatorname{div}_{x}(c p)$, with $p$ a Lagrange multiplier. However, this result can be quite deceptive: indeed the set of admissible test functions can be reduced to 0 . This is the case when the characteristic curves associated to the field $c$ escape to infinity (i.e. $\frac{\mathrm{d}}{\mathrm{d} t} X(t, x)=c(X(t, x))$ satisfies $X(t, x) \rightarrow \infty$ as $|x| \rightarrow \infty)$ : a test function $\varphi: \mathbb{R}^{N} \rightarrow \mathbb{R}$ such that $c \cdot \nabla_{x} \varphi=0$ is constant along these curves, hence it cannot have a compact support. The simplest example where the result is meaningless is given by $c=(1,0, \ldots, 0)$. Then the constraint $\operatorname{div}_{x}(c \rho)=0$ means $\partial_{1} \rho=0$ which actually implies $\rho=0$, since $\rho$ is a finite measure on $\mathbb{R}^{N}$.

\subsection{The purely oscillating case}

In this section, we shall see how the result of the previous section can be precised when we assume that the coefficients depend only on the fast variable $x / \varepsilon$. The flux is now a nonzero constant:

$$
c=\int_{Y} \int_{V} a(v) F(y, v) \mathrm{d} \mu(v) \mathrm{d} y \neq 0 .
$$


The idea is to follow the characteristic line with velocity $c / \varepsilon$. More precisely, the formal approach can be led by introducing the following Hilbert expansion:

$$
f_{\varepsilon}(t, x, v)=f^{0}\left(t, x-c \frac{t}{\varepsilon}, \frac{x}{\varepsilon}, v\right)+\varepsilon f^{1}\left(t, x-c \frac{t}{\varepsilon}, \frac{x}{\varepsilon}, v\right)+\varepsilon^{2} f^{2}\left(t, x-c \frac{t}{\varepsilon}, \frac{x}{\varepsilon}, v\right) \cdots
$$

Note that in this expansion, we only translate the macroscopic variable $x$, and leave the microscopic one, $y$, invariant. As a matter of fact, we expect the equilibrium flux $c$ to produce a drift at the macroscopic scale only.

Inserting this expansion in (1), a formal identification of the terms having the same power with respect to $\varepsilon$ leads to (compare with (5)):

$$
\begin{cases}a(v) \cdot \nabla_{y} f^{0}-Q\left(f^{0}\right)=0 & \left(\varepsilon^{-1} \text { terms }\right) \\ a(v) \cdot \nabla_{y} f^{1}-Q\left(f^{1}\right)=-(a(v)-c) \cdot \nabla_{x} f^{0} & \left(\varepsilon^{0} \text { terms }\right) \\ a(v) \cdot \nabla_{y} f^{2}-Q\left(f^{2}\right)=-\partial_{t} f^{0}-(a(v)-c) \cdot \nabla_{x} f^{1} & \left(\varepsilon^{1} \text { terms }\right) .\end{cases}
$$

We can now proceed as in Section 2:

- the first equation is the same as before, and yields: $f^{0}(t, x, y)=\rho(t, x) F(y, v)$, with $\rho(t, x)=\int_{V} \int_{Y} f^{0}(t, x, y, v) \mathrm{d} \mu(v) \mathrm{d} y$;

- then the first order equation becomes:

$$
\mathcal{T}\left(f^{1}\right)=-(a(v)-c) F(y, v) \cdot \nabla_{x} \rho(t, x) .
$$

The definition of $c$ and the normalization of $F$ provide the required solvability condition: There exists $\chi(y, v)$ solving

$$
\mathcal{T}(\chi)=-(a(v)-c) F(y, v),
$$

and we can define the first order corrector $f^{1}$ by (up to elements of $\operatorname{Ker}(\mathcal{T})$ )

$$
f^{1}(t, x, y, v)=\chi(y, v) \cdot \nabla_{x} \rho(t, x) .
$$

- Finally, the solvability condition for the second order corrector reads:

$$
\partial_{t} \rho+\operatorname{div}_{x} J=0
$$

with $J=\int_{Y} \int_{V}(a(v)-c) f^{1} \mathrm{~d} \mu(v) \mathrm{d} y$. In view of the previous expression of $f^{1}$, we deduce that

$$
J=-\widetilde{D} \nabla_{x} \rho
$$

with

$$
\widetilde{D}=-\int_{Y} \int_{V}(a(v)-c) \otimes \chi(y, v) \mathrm{d} \mu(v) \mathrm{d} y .
$$

Putting (22) and (23) together, we get

$$
\partial_{t} \rho(t, x)-\operatorname{div}_{x}\left[\widetilde{D} \nabla_{x} \rho(t, x)\right]=0
$$

In view of the Hilbert expansion, $f_{\varepsilon}$ asymptotically behaves as

$$
\rho\left(t, x-\frac{c}{\varepsilon} t\right) F\left(\frac{x}{\varepsilon}, v\right) .
$$


However, as in the previous section, we would like to study the asymptotic behaviour of $f_{\varepsilon}$ using only the natural $\mathrm{L}^{1}$ estimate. First of all, we set

$$
\widetilde{f}_{\varepsilon}(t, x, v)=f_{\varepsilon}(t, x+c t / \varepsilon, v),
$$

which satisfies

$$
\begin{cases}\partial_{t} \widetilde{f}_{\varepsilon}+\frac{1}{\varepsilon}(a(v)-c) \cdot \nabla_{x} \widetilde{f}_{\varepsilon}=\frac{1}{\varepsilon^{2}} \widetilde{Q}_{\varepsilon}\left(\widetilde{f}_{\varepsilon}\right) & \text { in }[0, \infty) \times \mathbb{R}^{N} \times V \\ \widetilde{f}_{\varepsilon}(0, x, v)=f_{\varepsilon}^{0}(x, v) & \text { in } \mathbb{R}^{N} \times V .\end{cases}
$$

The right-hand side is defined by

$$
\widetilde{Q}_{\varepsilon}\left(\widetilde{f}_{\varepsilon}\right)(t, x, v)=\int_{V} \sigma\left(x / \varepsilon+c t / \varepsilon^{2}, v, v^{\prime}\right) \widetilde{f}_{\varepsilon}\left(t, x, v^{\prime}\right) \mathrm{d} \mu\left(v^{\prime}\right)-\Sigma\left(x / \varepsilon+c t / \varepsilon^{2}, v\right) \widetilde{f}_{\varepsilon}(t, x, v) .
$$

This change of variable induces a new time scale: we are concerned with additional oscillations in time, with frequency $1 / \varepsilon^{2}$. Note that this time scaling is consistent with the diffusion scaling $\left(t \rightarrow t / \varepsilon^{2}, x \rightarrow x / \varepsilon\right)$. We then get the following theorem:

Theorem 5.2. Suppose (H1-H3) hold, where $\sigma\left(y, v, v^{\prime}\right)$ does not depend on the slow variable $x$. Let $F$ be the normalized solution of $\mathcal{T}(F)=0$ and set

$$
\int_{Y} \int_{V} a(v) F(y, v) \mathrm{d} \mu(v) \mathrm{d} y=c
$$

Suppose that $\rho_{\varepsilon}^{0}=\int_{V} f_{\varepsilon}^{0} \mathrm{~d} \mu(v)$ converges vaguely to $\rho^{0}$ in $\mathcal{M}^{1}\left(\mathbb{R}^{N}\right)$. Then, up to a subsequence, $\widetilde{\rho}_{\varepsilon}(t, x)=$ $\int_{V} \widetilde{f}_{\varepsilon} \mathrm{d} \mu(v)$, with $\widetilde{f}_{\varepsilon}$ defined by (24), converges to $\widetilde{\rho}$ vaguely, uniformly on any time interval $[0, T]$, and the limit satisfies the diffusion equation

$$
\partial_{t} \widetilde{\rho}-\operatorname{div}_{x}\left(\widetilde{D} \nabla_{x} \widetilde{\rho}\right)=0
$$

in $\mathcal{D}^{\prime}\left([0, \infty) \times \mathbb{R}^{N}\right)$ with Cauchy data $\rho^{0}$. The effective coefficients are defined by

$$
\widetilde{D}=-\int_{Y} \int_{V} \chi^{\star}(y, v) \otimes(a(v)-c) F(y, v) \mathrm{d} \mu(v) \mathrm{d} y,
$$

with $\chi^{\star}$ a solution to the cell problem $\mathcal{T}^{\star}\left(\chi^{\star}\right)=-(a(v)-c)$.

Remark 5.3. The symmetric part of the matrix $\widetilde{D}$ is positive definite. The proof is exactly the same as for $D$ in Proposition 3.6.

Proof. Consider the following simple remark. Let $\left\{e_{1}, \ldots, e_{N}\right\}$ be the canonical basis in $\mathbb{R}^{N}$. Let $\varphi$ be a $Y$-periodic function, i.e., for any $k \in \mathbb{Z}, \varphi\left(y+k e_{i}\right)=\varphi(y)$. To such a function we associate

$$
\begin{aligned}
\widetilde{\varphi}: \mathbb{R} \times \mathbb{R}^{N} & \longrightarrow \mathbb{R} \\
(\tau, y) & \longmapsto \widetilde{\varphi}(\tau, y)=\varphi(y+c \tau) .
\end{aligned}
$$

Let us introduce the vectors in $\mathbb{R}^{N+1}: \widetilde{e}_{0}=(1,-c), \widetilde{e}_{i}=\left(0, e_{i}\right), i \in\{1, \ldots, N\}$. Let $\tilde{Y}$ be the cube in $\mathbb{R}^{N+1}$ defined by the basis $\left\{\widetilde{e}_{0}, \ldots, \widetilde{e}_{N}\right\}$. Hence, $\widetilde{\varphi}$ is $\widetilde{Y}$-periodic, i.e., for any $k \in \mathbb{Z}, \widetilde{\varphi}\left((\tau, y)+k \widetilde{e}_{i}\right)=\widetilde{\varphi}(\tau, y)$. With this notation, $Q_{\varepsilon}$ corresponds to the operator

$$
\widetilde{Q}(f)(\tau, y)=\int_{V} \widetilde{\sigma}\left(\tau, y, v, v^{\prime}\right) f\left(v^{\prime}\right) \mathrm{d} \mu\left(v^{\prime}\right)-\widetilde{\Sigma}(\tau, y, v) f(v)
$$


evaluated at the fast variables $\tau=t / \varepsilon^{2}$ and $y=x / \varepsilon$, and the cross-section $\widetilde{\sigma}$ is $\tilde{Y}$-periodic. Then, we can reproduce all the previous arguments up to the following change of variables:

$$
\begin{cases}y \in Y \subset \mathbb{R}^{N} & \longrightarrow(\tau, y) \in \widetilde{Y} \subset \mathbb{R} \times \mathbb{R}^{N}, \\ v \in V & \longrightarrow\left(v_{0}, v\right) \in \widetilde{V}=\mathbb{R} \times V, \\ a(v) \in \mathbb{R}^{N} & \longrightarrow(a(v)-c) \in \mathbb{R}^{N}, \\ \mathrm{~d} \mu(v) & \longrightarrow \mathrm{d} \widetilde{\mu}\left(v_{0}, v\right)=\delta_{v_{0}=1} \otimes \mathrm{d} \mu(v) .\end{cases}
$$

In particular, $\widetilde{\mathcal{T}}=\partial_{\tau}+(a(v)-c) \cdot \nabla_{y}-\widetilde{Q}$ remains a Fredholm operator with index 0; its kernel is spanned by the positive function $F(y+c \tau, v)$. Accordingly, the double scale limit of $\tilde{f}_{\varepsilon}$ is given by $F(y+c \tau, v) \mathrm{d} \mu(v) \mathrm{d} y \mathrm{~d} \tau \widetilde{\rho}(t, \mathrm{~d} x) \mathrm{d} t$.

Remark 5.4. Returning to the original problem, the result tells us that $f_{\varepsilon}(t, x, v)$, the solution of $(1)$, behaves like $\widetilde{\rho}(t, x-c t / \varepsilon) F(x / \varepsilon, v)$.

\subsection{Parabolic scaling vs. hyperbolic scaling}

When the flux of the equilibrium function does not vanish, it is common to deal with another scaling, involving a slower time scale. Namely, we change $t$ to $\varepsilon t$ in (1), and we are interested in the equation

$$
\begin{cases}\partial_{t} \bar{f}_{\varepsilon}+a(v) \cdot \nabla_{x} \bar{f}_{\varepsilon}=\frac{1}{\varepsilon} Q_{\varepsilon}\left(\bar{f}_{\varepsilon}\right) & \text { in }[0, \infty) \times \mathbb{R}^{N} \times V, \\ \bar{f}_{\varepsilon \mid t=0}=f_{\varepsilon}^{0} & \text { in } \mathbb{R}^{N} \times V .\end{cases}
$$

The method of proof adopted in Theorem 3.11 leads to the following statement.

Theorem 5.5. Suppose (H1-H3) hold. Suppose that $\rho_{\varepsilon}^{0}=\int_{V} f_{\varepsilon}^{0} \mathrm{~d} \mu(v)$ converges vaguely to $\rho^{0}$ in $\mathcal{M}^{1}\left(\mathbb{R}^{N}\right)$. Then, up to a subsequence, $\bar{\rho}_{\varepsilon}(t, x)=\int_{V} \bar{f}_{\varepsilon} \mathrm{d} \mu(v)$ converges to $\bar{\rho} \geq 0$ vaguely, uniformly on any time interval $[0, T]$. The limit satisfies the transport equation

$$
\partial_{t} \bar{\rho}+\operatorname{div}_{x}(c \bar{\rho})=0
$$

in $\mathcal{D}^{\prime}\left([0, \infty) \times \mathbb{R}^{N}\right)$ with Cauchy data $\rho^{0}$.

Remark 5.6. When $c=0$ the limit equation reduces to $\partial_{t} \bar{\rho}=0$, which indicates that the hyperbolic scaling in (27) is meaningless: interesting effects should be observed on larger time scale, of order $1 / \varepsilon$, as shown in Section 4. We refer the reader to the lecture notes of Golse [24] for comments on this question. Similar results, starting from diffusion equations, have been described by Capdeboscq [11,12].

Remark 5.7. The result obtained in Section 5.2 can be interpreted as the description of a diffusive correction to the transport equation in Theorem 5.5. Indeed, Theorem 5.2 tells us that $\rho_{\varepsilon}(t, x)$ behaves like $\widetilde{\rho}(t, x-c t / \varepsilon)$, with $\widetilde{\rho}$ solution of $\partial_{t} \tilde{\rho}-\nabla_{x} \cdot\left(\widetilde{D} \nabla_{x} \widetilde{\rho}\right)=0$. Changing the time scale $t \rightarrow \varepsilon t$ means that $\bar{\rho}_{\varepsilon}(t, x)=\rho_{\varepsilon}(\varepsilon t, x)$ behaves like $r_{\varepsilon}(t, x)=\widetilde{\rho}(\varepsilon t, x-c t)$. This quantity satisfies the drift-diffusion equation

$$
\partial_{t} r_{\varepsilon}+c \cdot \nabla_{x} r_{\varepsilon}=\varepsilon \nabla_{x} \cdot\left(\widetilde{D} \nabla_{x} r_{\varepsilon}\right)
$$

with a diffusion coefficient of order $\varepsilon$. For approximation of kinetic equations, under a non-vanishing flux condition, by such an $\varepsilon$-dependent drift-diffusion equation, we refer the reader to Ringeisen and Sentis [41].

Proof. Multiplying $(27)$ by $\varphi(t, x, x / \varepsilon, v)$, we get

$$
\begin{aligned}
& \frac{\mathrm{d}}{\mathrm{d} t} \int_{\mathbb{R}^{N}} \int_{V} f_{\varepsilon} \varphi(t, x, x / \varepsilon, v) \mathrm{d} \mu(v) \mathrm{d} x=\int_{\mathbb{R}^{N}} \int_{V} f_{\varepsilon} \partial_{t} \varphi(t, x, x / \varepsilon, v) \mathrm{d} \mu(v) \mathrm{d} x \\
& +\int_{\mathbb{R}^{N}} \int_{V} f_{\varepsilon}\left(a(v) \cdot \nabla_{x} \varphi+\frac{1}{\varepsilon} a(v) \cdot \nabla_{y} \varphi+\frac{1}{\varepsilon} Q^{\star}(\varphi)\right)(t, x, x / \varepsilon, v) \mathrm{d} \mu(v) \mathrm{d} x
\end{aligned}
$$


(compare with (14)). We deduce that

$$
\int_{0}^{\infty} \int_{\mathbb{R}^{N}} \int_{V} f_{\varepsilon} \mathcal{T}^{\star}(\varphi)(t, x, x / \varepsilon, v) \mathrm{d} \mu(v) \mathrm{d} x \mathrm{~d} t \underset{\varepsilon \rightarrow 0}{\longrightarrow} 0
$$

holds. Next, we choose as test function $\varphi=\phi(t, x)+\varepsilon \psi(t, x, x / \varepsilon, v)$. We obtain

$$
\begin{array}{r}
\frac{\mathrm{d}}{\mathrm{d} t} \int_{\mathbb{R}^{N}} \int_{V} f_{\varepsilon}(\phi(t, x)+\varepsilon \psi(t, x, x / \varepsilon, v)) \mathrm{d} \mu(v) \mathrm{d} x=\int_{\mathbb{R}^{N}} \int_{V} f_{\varepsilon}\left(\partial_{t} \phi(t, x)+\varepsilon \partial_{t} \psi(t, x,, x / \varepsilon, v)\right) \mathrm{d} \mu(v) \mathrm{d} x \\
+\int_{\mathbb{R}^{N}} \int_{V} f_{\varepsilon}\left((a(v)-c) \cdot \nabla_{x} \phi(t, x)+\left(a(v) \cdot \nabla_{y} \psi+Q^{\star}(\psi)\right)(t, x, x / \varepsilon, v)\right) \mathrm{d} \mu(v) \mathrm{d} x \\
\quad+\int_{\mathbb{R}^{N}} \int_{V} f_{\varepsilon} c \cdot \nabla_{x} \phi(t, x) \mathrm{d} \mu(v) \mathrm{d} x+\int_{\mathbb{R}^{N}} \int_{V} f_{\varepsilon} \varepsilon a(v) \cdot \nabla_{x} \psi(t, x, x / \varepsilon, v) \mathrm{d} \mu(v) \mathrm{d} x .
\end{array}
$$

With $\psi=0$, we recover the conservation law

$$
\partial_{t} \rho_{\varepsilon}+\operatorname{div}_{x} J_{\varepsilon}=0
$$

where $J_{\varepsilon}(t, x)=\int_{V} a(v) f_{\varepsilon} \mathrm{d} \mu(v)$. We can define $\psi(t, x, y, v)$ which solves the cell problem $\mathcal{T}^{\star}(\psi)=(a(v)-c) \cdot \nabla_{x} \phi$, since the right hand side fulfils the compatibility condition. It amounts to saying that $\psi(t, x, y, v)=-\chi^{\star}(x, y, v) \cdot \nabla_{x} \phi$, with $\mathcal{T}^{\star}\left(\chi^{\star}\right)=-(a(v)-c)$.

Hence, we observe that

$$
\int_{\mathbb{R}^{N}} \int_{V} f_{\varepsilon} \phi \mathrm{d} \mu(v) \mathrm{d} x=\int_{\mathbb{R}^{N}} \rho_{\varepsilon} \phi \mathrm{d} x
$$

is equicontinuous on $[0, \infty)$. Therefore, we can suppose, up to a subsequence, that $\rho_{\varepsilon}$ converges to $\rho$ vaguely, uniformly on any interval $[0, T]$. Furthermore, passing to the limit in (30) yields the limit equation

$$
-\int_{\mathbb{R}^{N}} \phi(0, x) \rho^{0}(\mathrm{~d} x)=\int_{0}^{\infty} \int_{\mathbb{R}^{N}}\left(\partial_{t} \phi+c \cdot \nabla_{x} \phi\right)(t, x) \rho(t, \mathrm{~d} x) \mathrm{d} t
$$

for any test function in $\mathrm{C}_{c}^{\infty}\left([0, \infty) \times \mathbb{R}^{N}\right)$.

\section{Dissipation PRoperties}

In this section, we aim at showing that, in the situation we studied in Section 4, we can obtain better estimates on $f_{\varepsilon}$, when the equilibrium function $F(x, y, v)$ is bounded from below by a positive constant. In turn, the limit $\rho$ will be a function and not only a measure. In particular, we can observe regularizing effects due to the limit $\varepsilon \rightarrow 0$. These estimates are consequences of dissipation properties of the collision operator which has recently been studied in a much more general setting by Collet [15]. We refer the reader to his paper for deeper comments and nice applications of these properties.

\subsection{Entropies}

Our aim is to obtain some estimates on quantities, which could be called relative entropies, like

$$
\int_{\mathbb{R}^{N}} \int_{V} H(f / \Phi) \Phi \mathrm{d} \mu(v) \mathrm{d} x
$$

for convex functions $H$. 
Proposition 6.1. Let $f \in \mathrm{C}^{0}\left([0, T] ; \mathrm{L}^{1}\left(\mathbb{R}^{N} \times V\right)\right)$ solution of $\left(\partial_{t}+a(v) \cdot \nabla_{x}\right) f=Q(f)$, with $f_{\mid t=0}=f^{0}$. Let $\Phi$ be a positive function bounded from above and below, such that $\left(\partial_{t}+a(v) \cdot \nabla_{x}\right) \Phi \in \mathrm{L}^{\infty}\left((0, T) \times \mathbb{R}^{N} \times V\right)$. Let $H: \mathbb{R} \rightarrow \mathbb{R}$ be a nonnegative $\mathrm{C}^{1}$ convex function verifying $\left|s H^{\prime}(s)\right| \leq C H(s)$. Then, we have

$$
\begin{aligned}
\int_{\mathbb{R}^{N}} \int_{V} H(f / \Phi) \Phi(t) \mathrm{d} \mu(v) \mathrm{d} x+\int_{0}^{t} \int_{\mathbb{R}^{N}} \int_{V} D(f, \Phi) \mathrm{d} \mu(v) \mathrm{d} x \mathrm{~d} s & \int_{\mathbb{R}^{N}} \int_{V} H(f / \Phi) \Phi(0) \mathrm{d} \mu(v) \mathrm{d} x \\
& +\int_{0}^{t} \int_{\mathbb{R}^{N}} \int_{V} G(f / \Phi)\left(\partial_{t}+a(v) \cdot \nabla_{x}-Q\right) \Phi \mathrm{d} \mu(v) \mathrm{d} x \mathrm{~d} s,
\end{aligned}
$$

with $G(s)=H(s)-s H^{\prime}(s)$ and

$$
D(f, \Phi)=\int_{V} \sigma\left(v, v^{\prime}\right) \Phi\left(v^{\prime}\right)\left(H(f / \Phi)\left(v^{\prime}\right)-H(f / \Phi)(v)-H^{\prime}(f / \Phi)(v)\left(\frac{f}{\Phi}\left(v^{\prime}\right)-\frac{f}{\Phi}(v)\right)\right) \mathrm{d} \mu\left(v^{\prime}\right) .
$$

Considering smooth functions, we have

$$
\left(\partial_{t}+a(v) \cdot \nabla_{x}\right)[H(f / \Phi) \Phi]=H^{\prime}(f / \Phi)\left(\partial_{t}+a(v) \cdot \nabla_{x}\right) f+G(f / \Phi)\left(\partial_{t}+a(v) \cdot \nabla_{x}\right) \Phi .
$$

Integration of this relation leads to the result, when taking into account the following claim.

Lemma 6.2. Let $f \in \mathrm{L}^{1}(V)$, let $\Phi \in \mathrm{L}^{\infty}(V)$, with $\Phi>0$. Let $H: \mathbb{R} \rightarrow \mathbb{R}$ be a $\mathrm{C}^{1}$ function, verifying $H(s) \leq C s$. Then, the following relation

$$
Q(f) H^{\prime}(f / \Phi)=-D(f, \Phi)+Q(\Phi H(f / \Phi))-Q(\Phi) G(f / \Phi)
$$

holds with $G(s)$ and $D(f, \Phi)$ defined as in Proposition 6.1.

Remark 6.3. Considering convex functions $H$, we have $D(f, \Phi) \geq 0$. The restriction on the linear growth of $H$ is made only to guarantee integrability of the quantities under consideration. In particular, we obtain Proposition 6.1 for such a convex function. We get rid of $Q(\Phi H(f / \Phi))$ when integrating with respect to $v$, by the conservation property. To obtain the general case, it suffices to consider the approximation $H_{n}(s)=H(s) \chi_{s \leq n}+H^{\prime}(n)(s-n)+H(n) \chi_{s \geq n}$. By the Gronwall lemma, $\int_{\mathbb{R}^{N}} \int_{V} H_{n}(f / \Phi) \Phi \mathrm{d} \mu(v) \mathrm{d} x$, as well as the dissipation term, is bounded uniformly with respect to $n, t \in(0, T)$. We conclude by letting $n \rightarrow \infty$ and using the Fatou lemma.

Proof of Lemma 6.2. We will use the simple relation

$$
\int_{V} \sigma\left(v, v^{\prime}\right) g\left(v^{\prime}\right) \mathrm{d} \mu\left(v^{\prime}\right)=Q(g)+\int_{V} \sigma\left(v^{\prime}, v\right) \mathrm{d} \mu\left(v^{\prime}\right) g(v)
$$

for various functions $g$. First, we compute

$$
\begin{aligned}
Q(f) H^{\prime}(f / \Phi)(v) & =\left(\int_{V} \sigma\left(v, v^{\prime}\right) f\left(v^{\prime}\right) \mathrm{d} \mu\left(v^{\prime}\right)-\int_{V} \sigma\left(v^{\prime}, v\right) \mathrm{d} \mu\left(v^{\prime}\right) f(v)\right) H^{\prime}(f / \Phi)(v) \\
& =\left(\int_{V} \sigma\left(v, v^{\prime}\right) \Phi\left(v^{\prime}\right) \frac{f}{\Phi}\left(v^{\prime}\right) \mathrm{d} \mu\left(v^{\prime}\right)-\int_{V} \sigma\left(v^{\prime}, v\right) \Phi(v) \mathrm{d} \mu\left(v^{\prime}\right) \frac{f}{\Phi}(v)\right) H^{\prime}(f / \Phi)(v) \\
& =\int_{V} \sigma\left(v, v^{\prime}\right) \Phi\left(v^{\prime}\right) H^{\prime}(f / \Phi)(v)\left(\frac{f}{\Phi}\left(v^{\prime}\right)-\frac{f}{\Phi}(v)\right) \mathrm{d} \mu\left(v^{\prime}\right)+Q(\Phi) \frac{f}{\Phi} H^{\prime}(f / \Phi) .
\end{aligned}
$$


Since for a convex function $H$, we have $H(y)-H(x)-H^{\prime}(x)(y-x) \geq 0$, we would like to recognize in this expression the dissipation $D(f, \Phi)$ which is nonnegative for a convex function $H$. The missing term reads

$$
\begin{aligned}
\int_{V} \sigma\left(v, v^{\prime}\right) \Phi\left(v^{\prime}\right)\left(H(f / \Phi)(v)-H(f / \Phi)\left(v^{\prime}\right)\right) \mathrm{d} \mu\left(v^{\prime}\right)= & Q(\Phi) H(f / \Phi)(v)+\int_{V} \sigma\left(v^{\prime}, v\right) \mathrm{d} \mu\left(v^{\prime}\right) \Phi(v) H(f / \Phi)(v) \\
& -Q(\Phi H(f / \Phi))-\int_{V} \sigma\left(v^{\prime}, v\right) \mathrm{d} \mu\left(v^{\prime}\right) \Phi(v) H(f / \Phi)(v) \\
= & Q(\Phi) H(f / \Phi)(v)-Q(\Phi H(f / \Phi)) .
\end{aligned}
$$

Hence, we are led to (31). Note that similar computations have been performed for the quadratic case in the proof of Lemma 3.5.

\subsection{Application to the diffusion asymptotics}

We now apply Proposition 6.1 in the framework of Section 4, by taking into account the dependence with respect to $\varepsilon$ as follows:

$$
\left\{\begin{array}{l}
f \rightarrow f_{\varepsilon} \\
\partial_{t}+a(v) \cdot \nabla_{x} \rightarrow \partial_{t}+\varepsilon^{-1} a(v) \cdot \nabla_{x}, \quad Q \rightarrow \varepsilon^{-2} Q_{\varepsilon} \\
\Phi \rightarrow \Phi_{\varepsilon}(x, v)=F(x, x / \varepsilon, v)+\varepsilon \lambda(x, x / \varepsilon, v)
\end{array}\right.
$$

We recall that $\lambda$ is the solution of $a(v) \cdot \nabla_{y} \lambda-Q(\lambda)=-a(v) \cdot \nabla_{x} F$. Moreover, we shall assume the following hypotheses:

$$
\left\{\begin{array}{l}
\text { the functions } F(x, y, v) \text { and } \lambda(x, y, v) \text { are uniformly bounded, } \\
\quad \text { as well as their first derivatives with respect to } x \text {. } \\
\text { Moreover, } F(x, y, v) \text { is bounded from below by a positive constant. }
\end{array}\right.
$$

Since $F$ and $\lambda$ are continuous, with $F>0$, assumption (H4) holds for $x$ in any compact set $K$. Uniform bounds on the whole space are not easy to obtain. Nevertheless, this can be guaranteed by requiring a uniform behaviour of $\sigma$ at infinity (for instance the problem holds in the torus with periodic boundary conditions, or we impose that $\sigma$ tends to a constant at infinity...). As consequence of (H4), $\Phi_{\varepsilon}(x, v)$ will be bounded from above and below for $\varepsilon$ small enough.

Proposition 6.4. We assume (H1-H4) hold. Let $H$ be a nonnegative convex function such that there exists a constant $C$ satisfying, for any $s>0,\left|s H^{\prime}(s)\right| \leq C H(s)$. We set $\Phi_{\varepsilon}(x, v)=F(x, x / \varepsilon, v)+\varepsilon \lambda(x, x / \varepsilon, v)$ (which is bounded from above and below for $\varepsilon \in\left(0, \varepsilon_{0}\right)$ ). Suppose moreover that (H3) is strengthened by

$$
\sup _{0<\varepsilon<\varepsilon_{0}} \int_{\mathbb{R}^{N}} \int_{V} H\left(f_{\varepsilon}^{0} / \Phi_{\varepsilon}\right) \Phi_{\varepsilon} \mathrm{d} \mu(v) \mathrm{d} x \leq C_{0}<\infty .
$$

Then, the quantities

$$
\int_{\mathbb{R}^{N}} \int_{V} H\left(f_{\varepsilon} / \Phi_{\varepsilon}\right) \Phi_{\varepsilon} \mathrm{d} \mu(v) \mathrm{d} x \quad \text { and } \quad \frac{1}{\varepsilon^{2}} \int_{0}^{t} \int_{\mathbb{R}^{N}} D\left(f_{\varepsilon}, \Phi_{\varepsilon}\right) \mathrm{d} x \mathrm{~d} s
$$

are uniformly bounded with respect to $t \in[0, T]$, and $0<\varepsilon<\varepsilon_{0}$.

We recall that

$$
\begin{aligned}
\frac{1}{\varepsilon^{2}} \int_{0}^{t} \int_{\mathbb{R}^{N}} D\left(f_{\varepsilon}, \Phi_{\varepsilon}\right) \mathrm{d} x \mathrm{~d} s= & \frac{1}{\varepsilon^{2}} \int_{0}^{t} \int_{\mathbb{R}^{N}} \int_{V} \sigma\left(x, x / \varepsilon, v, v^{\prime}\right) \Phi_{\varepsilon}\left(v^{\prime}\right)\left(H\left(f_{\varepsilon} / \Phi_{\varepsilon}\right)(v)-H\left(f_{\varepsilon} / \Phi_{\varepsilon}\right)\left(v^{\prime}\right)\right. \\
& \left.-H^{\prime}\left(f_{\varepsilon} / \Phi_{\varepsilon}\right)(v)\left(f_{\varepsilon} / \Phi_{\varepsilon}\left(v^{\prime}\right)-f_{\varepsilon} / \Phi_{\varepsilon}(v)\right)\right) \mathrm{d} \mu(v) \mathrm{d} x \mathrm{~d} s \geq 0 .
\end{aligned}
$$


Proof. Integration of the equation satisfied by $\left(\partial_{t}+\varepsilon^{-1} a(v) \cdot \nabla_{x}\right)\left[H\left(f_{\varepsilon} / \phi_{\varepsilon}\right) \Phi_{\varepsilon}\right]$ gives, according to Proposition 6.1 ,

$$
\begin{aligned}
\frac{\mathrm{d}}{\mathrm{d} t} \int_{\mathbb{R}^{N}} \int_{V} H\left(f_{\varepsilon} / \Phi_{\varepsilon}\right) \Phi_{\varepsilon} \mathrm{d} \mu(v) \mathrm{d} x+\frac{1}{\varepsilon^{2}} \int_{\mathbb{R}^{N}} D\left(f_{\varepsilon}, \Phi_{\varepsilon}\right) \mathrm{d} x & \\
& \leq \int_{\mathbb{R}^{N}} \int_{V} G\left(f_{\varepsilon} / \Phi_{\varepsilon}\right)\left(\frac{1}{\varepsilon} a(v) \cdot \nabla_{x}-\frac{1}{\varepsilon^{2}} Q_{\varepsilon}\right)\left(\Phi_{\varepsilon}\right) \mathrm{d} \mu(v) \mathrm{d} x
\end{aligned}
$$

However, we have

$$
\begin{aligned}
\left(\frac{1}{\varepsilon} a(v) \cdot \nabla_{x}-\frac{1}{\varepsilon^{2}} Q_{\varepsilon}\right)[\Phi(x, x / \varepsilon, v)]= & \frac{1}{\varepsilon^{2}}\left(a(v) \cdot \nabla_{y} F-Q(F)\right)(x, x / \varepsilon, v) \\
& +\frac{1}{\varepsilon}\left(a(v) \cdot \nabla_{y} \lambda-Q(\lambda)+a(v) \cdot \nabla_{x} F\right)(x, x / \varepsilon, v)+a(v) \cdot \nabla_{x} \lambda(x, x / \varepsilon, v) \\
= & a(v) \cdot \nabla_{x} \lambda(x, x / \varepsilon, v)
\end{aligned}
$$

which is bounded, uniformly with respect to $\varepsilon$. Hence, we get

$$
\frac{\mathrm{d}}{\mathrm{d} t} \int_{\mathbb{R}^{N}} \int_{V} H\left(f_{\varepsilon} / \Phi_{\varepsilon}\right) \Phi_{\varepsilon} \mathrm{d} \mu(v) \mathrm{d} x+\int_{\mathbb{R}^{N}} D\left(f_{\varepsilon}, \Phi_{\varepsilon}\right) \mathrm{d} x \leq C \int_{\mathbb{R}^{N}} \int_{V} H\left(f_{\varepsilon} / \Phi_{\varepsilon}\right) \Phi_{\varepsilon} \mathrm{d} \mu(v) \mathrm{d} x,
$$

which allows us to end by applying the Gronwall lemma.

We are now going to use this statement with $H(s)=s^{2} / 2$, to improve the regularity of the limit $\rho$ obtained in Theorem 3.11; in turn, we obtain uniqueness of the limit.

Corollary 6.5. Let the assumptions of Proposition 6.4 hold, with $H(s)=s^{2} / 2$. Suppose moreover that $\chi^{\star}$ is uniformly bounded, as well as its first derivatives. Then, the whole sequence $\rho_{\varepsilon}(t, x)=\int_{V} f_{\varepsilon} \mathrm{d} \mu(v)$ converges to $\rho$ in $\mathrm{C}^{0}\left([0, T] ; \mathrm{L}^{2}\left(\mathbb{R}^{N}\right)\right.$-weakly). The limit is the unique solution in $\mathrm{L}^{\infty}\left(0, T ; \mathrm{L}^{2}\left(\mathbb{R}^{N}\right)\right) \cap \mathrm{L}^{2}\left(0, T ; \mathrm{H}^{1}\left(\mathbb{R}^{N}\right)\right)$ of the drift-diffusion equation $\partial_{t} \rho-\operatorname{div}_{x}\left(D \nabla_{x} \rho-U \rho\right)=0$.

The keypoint is to justify the regularity of the limit.

Lemma 6.6. The limit density $\rho$ belongs to $L^{2}\left(0, T ; H^{1}\left(\mathbb{R}^{N}\right)\right)$.

Since we have uniqueness of the solution of the parabolic equation in this class of functions, the whole sequence $\rho_{\varepsilon}$ converges to a unique cluster point. Lemma 6.6 illustrates the regularizing effects due to the diffusion approximation: the limit $\rho$ has much more regularity than can be expected from the bounds on $\rho_{\varepsilon}$. We divide the proof into several steps.

\section{Step 1: Expansion of the Solution.}

We recall that $\Phi_{\varepsilon}(x, v)=F(x, x / \varepsilon, v)+\varepsilon \lambda(x, x / \varepsilon, v)$ is bounded from above and below for $\varepsilon \in\left(0, \varepsilon_{0}\right)$. We expand the solution as follows

$$
\left\{\begin{array}{l}
f_{\varepsilon}(t, x, v)=r_{\varepsilon}(t, x) \Phi_{\varepsilon}(x, v)+\varepsilon g_{\varepsilon}(t, x, v), \\
r_{\varepsilon}(t, x)=\int_{V} \frac{f_{\varepsilon}}{\Phi_{\varepsilon}} \mathrm{d} \mu(v), \\
\int_{V} \frac{g_{\varepsilon}}{\Phi_{\varepsilon}} \mathrm{d} \mu(v)=0
\end{array}\right.
$$

By Proposition 6.4,

so that

$$
f_{\varepsilon} \text { is bounded in } \mathrm{L}^{\infty}\left(0, T ; \mathrm{L}^{2}\left(\mathbb{R}^{N} \times V\right)\right),
$$

$$
\rho_{\varepsilon} \text { and } r_{\varepsilon} \text { are bounded in } \mathrm{L}^{\infty}\left(0, T ; \mathrm{L}^{2}\left(\mathbb{R}^{N}\right)\right) \text {. }
$$


On the other hand, the dissipation term controls the quantity

$$
\frac{1}{\varepsilon^{2}} \int_{V} \int_{V}\left|\frac{f_{\varepsilon}}{\Phi_{\varepsilon}}\left(v^{\prime}\right)-\frac{f_{\varepsilon}}{\Phi_{\varepsilon}}(v)\right|^{2} \mathrm{~d} \mu\left(v^{\prime}\right) \mathrm{d} \mu(v)=\int_{V} \int_{V}\left|\frac{g_{\varepsilon}}{\Phi_{\varepsilon}}\left(v^{\prime}\right)-\frac{g_{\varepsilon}}{\Phi_{\varepsilon}}(v)\right|^{2} \mathrm{~d} \mu\left(v^{\prime}\right) \mathrm{d} \mu(v)=2 \int_{V}\left|\frac{g_{\varepsilon}}{\Phi_{\varepsilon}}\left(v^{\prime}\right)\right|^{2} \mathrm{~d} \mu(v) .
$$

We deduce that

$$
g_{\varepsilon} \text { is bounded in } \mathrm{L}^{2}\left((0, T) \times \mathbb{R}^{N} \times V\right) .
$$

However, it is worth pointing out that we cannot deduce a $\mathrm{L}^{2}$ estimate on the current

$$
J_{\varepsilon}(t, x)=\int_{V} \frac{a(v)}{\varepsilon} f_{\varepsilon} \mathrm{d} \mu(v)=\frac{1}{\varepsilon} r_{\varepsilon}(t, x) \int_{V} a(v) \Phi_{\varepsilon} \mathrm{d} \mu(v)+\int_{V} a(v) g_{\varepsilon} \mathrm{d} \mu(v) .
$$

This is unusual in diffusion approximation and homogenization and can be an obstacle to the strong convergence of $\rho_{\varepsilon}$.

Combining the estimates provided by Proposition 6.4, and the results obtained in Theorem 3.11, we have, at least for a subsequence,

$$
\left\{\begin{array}{l}
\left.\rho_{\varepsilon}(t, x) \rightarrow \rho(t, x) \quad \text { in } \mathrm{C}^{0}\left([0, T] ; \mathrm{L}^{2}\left(\mathbb{R}^{N}\right)-\text { weak }\right)\right) \\
f_{\varepsilon}(t, x, v) \rightarrow \rho(t, x) F(x, y, v) \quad \text { in the (usual) double-scale sense. }
\end{array}\right.
$$

Furthermore, $\Phi_{\varepsilon}$ is an admissible function (see [1]) and converges double-scale strongly to $F(x, y, v)$. Therefore, identifying limits leads to

$$
r_{\varepsilon} \rightarrow \rho \quad \text { weakly in } \mathrm{L}^{\infty}\left(0, T ; \mathrm{L}^{2}\left(\mathbb{R}^{N}\right)\right) \text { and double scale }
$$

(in particular, note that the double scale limit of $r_{\varepsilon}$, in $\mathrm{L}^{2}\left((0, T) \times \mathbb{R}^{N} \times Y\right)$, does not depend on the periodic variable $y \in Y)$.

\section{Step 2: Limit Current.}

Even if we have no immediate estimate in a reasonable functional space, we can identify the limit current, at least in the sense of distributions.

Lemma 6.7. We can write the current as

$$
J_{\varepsilon}=\varepsilon \partial_{t} E_{\varepsilon}+K_{\varepsilon}
$$

with $E_{\varepsilon}$ bounded in $\mathrm{L}^{\infty}\left(0, T ; \mathrm{L}^{2}\left(\mathbb{R}^{N}\right)\right), K_{\varepsilon}$ bounded in $\mathrm{L}^{\infty}\left(0, T ; \mathrm{H}^{-1}\left(\mathbb{R}^{N}\right)\right)$. Letting $\varepsilon \rightarrow 0$ yields

$$
J_{\varepsilon} \underset{\varepsilon \rightarrow 0}{\longrightarrow}-D \nabla_{x} \rho+U \rho \quad \text { in } \mathcal{D}^{\prime}\left((0, T) \times \mathbb{R}^{N}\right) .
$$

Proof. Let $\Psi \in\left(\mathrm{C}_{0}^{\infty}\left((0, T) \times \mathbb{R}^{N}\right)\right)^{N}$. We associate to $\Psi$, the following function

$$
X(t, x, y, v)=\chi^{\star}(x, y, v) \cdot \Psi(t, x),
$$


which is a continuous function of its arguments, as its first derivative $\partial_{x} X$. It satisfies $a(v) \cdot \nabla_{y} X+Q^{\star}(X)=a(v) \cdot \Psi$. Then, we get (see (14) with $\varphi=\varepsilon X$ )

$$
\begin{aligned}
\int_{0}^{T} \int_{\mathbb{R}^{N}} J_{\varepsilon} \cdot \Psi \mathrm{d} x \mathrm{~d} t= & \int_{0}^{T} \int_{\mathbb{R}^{N}} \int_{V} \frac{a(v)}{\varepsilon} \cdot \Psi f_{\varepsilon} \mathrm{d} \mu(v) \mathrm{d} x \mathrm{~d} t \\
= & \frac{1}{\varepsilon} \int_{0}^{T} \int_{\mathbb{R}^{N}} \int_{V}\left(a(v) \cdot \nabla_{y} X+Q^{\star}(X)\right)(t, x, x / \varepsilon, v) f_{\varepsilon} \mathrm{d} \mu(v) \mathrm{d} x \mathrm{~d} t \\
= & -\varepsilon \int_{0}^{T} \int_{\mathbb{R}^{N}} \int_{V} \partial_{t} X(t, x, x / \varepsilon, v) f_{\varepsilon} \mathrm{d} \mu(v) \mathrm{d} x \mathrm{~d} t \\
& -\int_{0}^{T} \int_{\mathbb{R}^{N}} \int_{V}\left(a(v) \cdot \nabla_{x} X\right)(t, x, x / \varepsilon, v) f_{\varepsilon} \mathrm{d} \mu(v) \mathrm{d} x \mathrm{~d} t .
\end{aligned}
$$

The first term reads

$$
-\varepsilon \int_{0}^{T} \int_{\mathbb{R}^{N}}\left(\int_{V} f_{\varepsilon} \chi^{\star}(x, x / \varepsilon, v) \mathrm{d} \mu(v)\right) \cdot \partial_{t} \Psi \mathrm{d} x \mathrm{~d} t=\varepsilon\left\langle\partial_{t} E_{\varepsilon}, \Psi\right\rangle
$$

with

$$
E_{\varepsilon}(t, x)=\int_{V} f_{\varepsilon} \chi^{\star}(x, x / \varepsilon, v) \mathrm{d} \mu(v)
$$

bounded in $\mathrm{L}^{\infty}\left(0, T ; \mathrm{L}^{2}\left(\mathbb{R}^{N}\right)\right)$. The second term reads

$$
\begin{aligned}
\left\langle K_{\varepsilon}, \Psi\right\rangle= & \int_{0}^{T} \int_{\mathbb{R}^{N}}\left(\int_{V}\left(D_{x} \chi^{\star}\right)^{T}(x, x / \varepsilon, v) a(v) f_{\varepsilon} \mathrm{d} \mu(v)\right) \cdot \Psi \mathrm{d} x \mathrm{~d} t \\
& +\int_{0}^{T} \int_{\mathbb{R}^{N}}\left(\int_{V} a(v) \otimes \chi^{\star}(x, x / \varepsilon, v) f_{\varepsilon} \mathrm{d} \mu(v)\right): D_{x} \Psi \mathrm{d} x \mathrm{~d} t
\end{aligned}
$$

which immediately gives the aforementioned bound.

Moreover, passing to the limit yields

$$
\begin{aligned}
\left\langle J_{\varepsilon}, \Psi\right\rangle \underset{\varepsilon \rightarrow 0}{\longrightarrow} & 0+\int_{0}^{T} \int_{\mathbb{R}^{N}}\left(\int_{Y} \int_{V}\left(D_{x} \chi^{\star}\right)^{T} a(v) F \mathrm{~d} \mu(v) \mathrm{d} y\right) \rho(t, x) \cdot \Psi \mathrm{d} x \mathrm{~d} t \\
& +\int_{0}^{T} \int_{\mathbb{R}^{N}}\left(\int_{Y} \int_{V} a(v) \otimes \chi^{\star} F \mathrm{~d} \mu(v) \mathrm{d} y\right) \rho: D_{x} \Psi \mathrm{d} x \mathrm{~d} t \\
\underset{\varepsilon \rightarrow 0}{\longrightarrow} & \int_{0}^{T} \int_{\mathbb{R}^{N}}\left(\operatorname{div}_{x}\left(D^{T} \Psi\right)+U \cdot \psi\right) \rho \mathrm{d} x \mathrm{~d} t=\left\langle-D \nabla_{x} \rho+U \rho, \Psi\right\rangle .
\end{aligned}
$$

Step 3: Regularity of the Limit Macroscopic Density.

Lemma 6.6 follows from the following claim.

Lemma 6.8. The limit of $\nabla_{x} r_{\varepsilon}$ in $\mathcal{D}^{\prime}\left((0, T) \times \mathbb{R}^{N}\right)$ belongs to $\mathrm{L}^{2}\left((0, T) \times \mathbb{R}^{N}\right)$.

Proof. Let us write the equation satisfied by $J_{\varepsilon}$. Multiplying $f_{\varepsilon}$ by $a(v)$ and integrating yields

$$
\varepsilon^{2} \partial_{t} J_{\varepsilon}+\operatorname{Div}_{x} \mathbb{P}_{\varepsilon}=\int_{V} \frac{a(v)}{\varepsilon} Q_{\varepsilon}\left(f_{\varepsilon}\right) \mathrm{d} \mu(v)
$$


which involves the second moment of $f_{\varepsilon}$

$$
\mathbb{P}_{\varepsilon}(t, x)=\int_{V} a(v) \otimes a(v) f_{\varepsilon} \mathrm{d} \mu(v) .
$$

The kinetic pressure reads

$$
\begin{aligned}
\operatorname{Div}_{x} \mathbb{P}_{\varepsilon}= & r_{\varepsilon} \int_{V} a(v) a(v) \cdot\left(\frac{1}{\varepsilon} \nabla_{y} F+\nabla_{x} F+\nabla_{y} \lambda+\varepsilon \nabla_{x} \lambda\right)(x, x / \varepsilon, v) \mathrm{d} \mu(v) \\
& +\Theta_{\varepsilon} \nabla_{x} r_{\varepsilon}+\varepsilon \operatorname{Div}_{x} \mathbb{Q}_{\varepsilon}
\end{aligned}
$$

with the matrices

$$
\left\{\begin{array}{l}
\Theta_{\varepsilon}(x)=\Theta(x, x / \varepsilon)=\int_{V} a(v) \otimes a(v)(F+\varepsilon \lambda)(x, x / \varepsilon, v) \mathrm{d} \mu(v) \\
\mathbb{Q}_{\varepsilon}(t, x)=\int_{V} a(v) \otimes a(v) g_{\varepsilon} \mathrm{d} \mu(v),
\end{array}\right.
$$

while the right-hand side in (33) becomes

$$
\frac{1}{\varepsilon} r_{\varepsilon} \int_{V} a(v) Q(F+\varepsilon \lambda)(x, x / \varepsilon, v) \mathrm{d} \mu(v)+\int_{V} a(v) Q_{\varepsilon}\left(g_{\varepsilon}\right) \mathrm{d} \mu(v) .
$$

Define

$$
S_{\varepsilon}(t, x)=\int_{V} a(v) Q_{\varepsilon}\left(g_{\varepsilon}\right) \mathrm{d} \mu(v)-\varepsilon r_{\varepsilon} \int_{V} a(v) a(v) \cdot \nabla_{x} \lambda(x, x / \varepsilon, v) \mathrm{d} \mu(v) .
$$

This quantity is bounded in $\mathrm{L}^{2}\left((0, T) \times \mathbb{R}^{N}\right)$, as well as $\mathbb{Q}_{\varepsilon}$. On the other hand, the matrix $\Theta_{\varepsilon}$ can be evaluated uniformly: there exists $\theta_{*}, \theta^{*}$ such that

$$
\text { for any } \xi \in \mathbb{R}^{N}, 0<\theta_{*}|\xi|^{2} \leq \Theta_{\varepsilon}(x) \xi \cdot \xi \leq \theta^{*}|\xi|^{2}, \quad\left\|D_{x} \Theta_{\varepsilon}\right\|_{L^{\infty}\left(\mathbb{R}^{N}\right)} \leq \frac{\theta^{*}}{\varepsilon} .
$$

Therefore, equation (33) can be rewritten

$$
\begin{aligned}
\nabla_{x} r_{\varepsilon} & =\Theta_{\varepsilon}^{-1}\left(S_{\varepsilon}-\varepsilon^{2} \partial_{t} J_{\varepsilon}-\varepsilon \operatorname{Div}_{x} \mathbb{Q}_{\varepsilon}\right) \\
& =\Theta_{\varepsilon}^{-1} S_{\varepsilon}-\varepsilon^{3} \partial_{t}^{2}\left(\Theta_{\varepsilon}^{-1} E_{\varepsilon}\right)-\varepsilon \partial_{t}\left(\varepsilon \Theta_{\varepsilon}^{-1} K_{\varepsilon}\right)-\varepsilon \Theta_{\varepsilon}^{-1} \operatorname{Div}_{x} \mathbb{Q}_{\varepsilon}
\end{aligned}
$$

using Lemma 6.7. It tends to $\nabla_{x} r=\nabla_{x} \rho$ in $\mathcal{D}^{\prime}\left((0, T) \times \mathbb{R}^{N}\right)$. Let $\varphi \in \mathrm{C}_{c}^{\infty}\left((0, T) \times \mathbb{R}^{N}\right)$. We can estimate as follows:

$$
\begin{aligned}
\left|\left\langle\nabla_{x} r_{\varepsilon}, \varphi\right\rangle\right| \leq & \left\|\Theta_{\varepsilon}^{-1} S_{\varepsilon}\right\|_{\mathrm{L}_{t x}^{2}}\|\varphi\|_{\mathrm{L}_{t x}^{2}}+\varepsilon^{3}\left\|\Theta_{\varepsilon}^{-1} E_{\varepsilon}\right\|_{\mathrm{L}_{t x}^{2}}\left\|\partial_{t}^{2} \varphi\right\|_{\mathrm{L}_{t x}^{2}} \\
& +\varepsilon\left\|K_{\varepsilon}\right\|_{\mathrm{L}_{t}^{2}\left(\mathrm{H}_{x}^{-1}\right)}\left\|\varepsilon\left(\Theta_{\varepsilon}^{-1}\right)^{T} \partial_{t} \varphi\right\|_{\mathrm{L}_{t}^{2}\left(\mathrm{H}_{x}^{1}\right)}+\left\|\mathbb{Q}_{\varepsilon}\right\|_{\mathrm{L}_{t x}^{2}}\left\|\varepsilon D_{x}\left(\left(\Theta_{\varepsilon}^{-1}\right)^{T} \varphi\right)\right\|_{\mathrm{L}_{t x}^{2}} \\
\leq & C\left(\|\varphi\|_{\mathrm{L}_{t x}^{2}}+\varepsilon^{3}\left\|\partial_{t}^{2} \varphi\right\|_{\mathrm{L}_{t x}^{2}}+\varepsilon\left\|\partial_{t}^{2} \varphi\right\|_{\mathrm{L}_{t}^{2}\left(\mathrm{H}_{x}^{1}\right)}++\varepsilon\left\|D_{x} \varphi\right\|_{\mathrm{L}_{t x}^{2}}\right)
\end{aligned}
$$

where the constant $C$ does not depend on $\varepsilon$. Therefore, we deduce that

$$
\lim _{\varepsilon \rightarrow 0}\left|\left\langle\nabla_{x} r_{\varepsilon}, \varphi\right\rangle\right| \leq C\|\varphi\|_{L_{t x}^{2}}
$$

which ends the proof. 


\section{Appendix A. Resolution of the Cell problems}

This Appendix is devoted to the proofs of the statements on the cell problems in Section 3.2.

Proof of Proposition 3.1. As a preliminary, we remark that, since $\sigma \in \mathrm{L}_{\#}^{\infty}(Y \times V \times V), Q$ is a bounded operator on $\mathrm{L}_{\#}^{p}(Y \times V)$ for any $1 \leq p \leq \infty$. We also note that $\int_{Y} \int_{V} \mathcal{T}(f) \mathrm{d} \mu(v) \mathrm{d} y=0$ and $\mathbb{1} \in \operatorname{Ker}\left(\mathcal{T}^{\star}\right)$. We wish to determine the dimension of $\operatorname{Ker}\left(\mathcal{T}^{\star}\right)$ and to conclude by a Fredholm alternative argument. Let us first rewrite $\mathcal{T}$ as a perturbation of $\mathcal{A}=a(v) \cdot \nabla_{y}+\Sigma(y, v)$ by an integral operator $K$

$$
\left\{\begin{array}{l}
\mathcal{T}=\mathcal{A}-K \\
K(f)=\int_{V} \sigma\left(y, v, v^{\prime}\right) f\left(y, v^{\prime}\right) \mathrm{d} \mu\left(v^{\prime}\right)
\end{array}\right.
$$

The advection operator $\mathcal{A}$ is invertible. Indeed, integrating along the characteristics lines $y+\sigma v$ the equation $\mathcal{A} f=h$, we get

$$
f(y, v)=\mathcal{A}^{-1} h=\int_{0}^{\infty} \exp \left(-\int_{0}^{\sigma} \Sigma(y-\tau a(v), v) \mathrm{d} \tau\right) h(y-\sigma a(v), v) \mathrm{d} \sigma .
$$

Since $\Sigma(y, v) \geq \mu(V) \sigma_{*}>0$, it defines a bounded operator on $\mathrm{L}_{\#}^{p}(Y \times V)$. We also notice that $\mathcal{A}^{-1}$ is a nonnegative operator: if $h \geq 0$ (resp., $h>0$ ), then $f=\mathcal{A}^{-1} h \geq 0$ (resp., $>0$ ). Now, we rewrite the equation $\mathcal{T}(f)=g$ as

$$
\left(I-K \circ \mathcal{A}^{-1}\right) h=g, \quad h=\mathcal{A} f .
$$

The key of the proof relies on the following claim:

Lemma A.1. Suppose (H1), and let $\sigma \in \mathrm{C}^{0}\left(Y ; \mathrm{L}^{\infty}(V \times V)\right)$, with $0<\sigma_{*} \leq \sigma \leq \sigma^{*}$. Then the operator $\mathcal{Q}=K \circ \mathcal{A}^{-1}$ is compact on $\mathrm{L}_{\#}^{p}(Y \times V)$ for $1<p<\infty$.

Let us temporarily assume this lemma. Since $\sigma>0$, for any nonnegative function $f$, we have $K(f)>0$. Hence, $\mathcal{Q}=K \circ \mathcal{A}^{-1}$ is a positive, compact operator on $\mathrm{L}_{\#}^{p}(Y \times V)$, and the Krein-Rutman theorem applies (see [31]): the spectral radius, $\lambda$, is an eigenvalue, associated to a non negative eigenfunction $H \geq 0$. We set $\mathcal{A} F=H$, so that $K(F)=\lambda \mathcal{A} F$. By using (4) and the periodicity with respect to $y$, integration of this relation leads to

$$
\lambda \int_{Y} \int_{V} \Sigma F \mathrm{~d} \mu(v) \mathrm{d} y=\int_{Y} \int_{V} \Sigma F \mathrm{~d} \mu(v) \mathrm{d} y>0 .
$$

We deduce that $\lambda=1$ is the principal eigenvalue of $\mathcal{Q}$. Furthermore, from $F=\mathcal{A}^{-1} H \geq 0$, we deduce that $\mathcal{A} F=H=K(F)>0$; hence, $F=\mathcal{A}^{-1} H>0$. Finally, since $f \geq 0$ implies $\mathcal{Q}(f)>0$, we deduce that the dimension of the eigenspace is one. Similar arguments apply to the adjoint operator. This ends the proof of $i)$.

Next, we apply the Fredholm alternative: $\mathcal{T}(f)=g$ is solvable for $g \in \operatorname{Ran}(\mathcal{T})=\operatorname{Ker}\left(\mathcal{T}^{\star}\right)^{\perp}$. Since eigenspaces of $\mathcal{T}$ and $\mathcal{T}^{\star}$ are spanned by positive functions, the condition of vanishing integral guarantees uniqueness. Hence, for any elements in $\mathrm{L}_{\#, 0}^{p}=\left\{g \in \mathrm{L}_{\#}^{p}(Y \times V), \int_{Y} \int_{V} g \mathrm{~d} \mu(v) \mathrm{d} y=0\right\}$, we find a unique $f \in \mathrm{L}_{\#, 0}^{p}$ solution of $\mathcal{T}(f)=g$. The Open Mapping Theorem (see, for instance [10], p. 19) gives the existence of $C>0$ such that $\|f\|_{\mathrm{L}_{\#}^{p}} \leq C\|g\|_{\mathrm{L}_{\#}^{p}}$. The same conclusion applies for the adjoint operator.

Proof of Lemma A.1. This result is by now quite classical, as a consequence of the Averaging lemma (see, for instance [16], Chap. XXI.5). We recall the main steps of the proof for the sake of completeness. First, we split $Y$ into $h^{N}$ cubes of size $h>0$, denoted by $E_{h}^{i}, i \in\left\{1, \ldots, h^{N}\right\}$. and we replace $K$ by

$$
K_{h}(f)=\sum_{i=1}^{h^{N}} \chi_{E_{h}^{i}}(y) \int_{V}\left(\frac{1}{\left|E_{h}^{i}\right|} \int_{E_{h}^{i}} \sigma\left(z, v, v^{\prime}\right) \mathrm{d} z\right) f\left(y, v^{\prime}\right) \mathrm{d} \mu\left(v^{\prime}\right)
$$


Since $\sigma \in \mathrm{C}^{0}\left(Y ; \mathrm{L}^{\infty}(V \times V)\right)$, we have $\left\|K-K_{h}\right\|_{\mathcal{L}\left(\mathrm{L}^{p}\right)} \rightarrow 0$ as $h$ tends to 0 . Hence, we are left with the task of proving the compactness of $K_{h} \circ \mathcal{A}^{-1}$. This a consequence of the compactness for an operator $K$ whose kernel $\sigma$ depends only on the velocity variables $v, v^{\prime}$.

Actually, the problem reduces to consider a continuous kernel. Indeed, we approximate $\sigma \in \mathrm{L}^{p}\left(V ; L^{p^{\prime}}(V)\right)$ by continuous functions $\sigma_{n}$ and we have

$$
\left\|\left(K_{n}-K\right) f\right\|_{\mathrm{L}^{p}(Y \times V)}^{p} \leq\|f\|_{\mathrm{L}^{p}(Y \times V)}^{p}\left\|\sigma_{n}-\sigma\right\|_{\mathrm{L}^{p}\left(V ; L^{p^{\prime}}(V)\right)}^{p},
$$

i.e. $\left\|K-K_{n}\right\|_{\mathcal{L}\left(\mathrm{L}^{p}\right)} \rightarrow 0$ as $n$ tends to $\infty$.

Thus, let $\sigma\left(v, v^{\prime}\right) \in \mathrm{C}^{0}\left(V ; L^{p^{\prime}}(V)\right)$. By applying the $\mathrm{L}^{p}$ version of the average lemma (see $\left.[18,25]\right)$, we obtain that, for $v \in V$ fixed, the mapping

$$
g \in \mathrm{L}^{p}(Y \times V) \longmapsto \int_{V} \sigma\left(v, v^{\prime}\right) \mathcal{A}^{-1} g\left(y, v^{\prime}\right) \mathrm{d} \mu\left(v^{\prime}\right)
$$

has its range in a Sobolev space $\mathrm{W}^{s, p}(Y), s>0$ (precisely $s=\gamma \inf (1 / p, 1-1 / p)$ ), and is therefore compact in $\mathrm{L}^{p}(Y)$. But we also have

$$
\int_{Y}|K f(y, v+h)-K f(y, v)|^{p} \mathrm{~d} y \leq\|f\|_{\mathrm{L}^{p}(Y \times V)}^{p}\left(\int_{V}\left|\sigma\left(v+h, v^{\prime}\right)-\sigma\left(v, v^{\prime}\right)\right|^{p^{\prime}} \mathrm{d} \mu\left(v^{\prime}\right)\right)^{p / p^{\prime}} .
$$

Hence, we conclude by the Arzela-Ascoli theorem that $g \mapsto K \circ A^{-1} g\left(y, v^{\prime}\right)$ is compact in $\mathrm{C}^{0}\left(V ; \mathrm{L}^{p}(Y)\right)$, thus in $\mathrm{L}^{p}(Y \times V)$.

Proof of Lemma 3.2. When $\sigma$ and $g$ have the regularity of Lemma 3.2, the previous proof shows that the solution $h(y, v)$ of

$$
\left(I-K \circ \mathcal{A}^{-1}\right) h=g,
$$

actually belongs to $\mathrm{C}^{0}\left(V ; \mathrm{L}^{p}(Y)\right)$ (since $K \circ \mathcal{A}^{-1} h$ has its range in $\mathrm{C}^{0}\left(V ; \mathrm{L}^{p}(Y)\right)$ ). It remains to see that $f(y, v)=\mathcal{A}^{-1} h$ has the same regularity. But this is an immediate consequence of the following formula:

$$
f(y, v)=\int_{0}^{\infty} \exp \left(-\int_{0}^{\sigma} \Sigma(y-\tau a(v), v) \mathrm{d} \tau\right) h(y-\sigma a(v), v) \mathrm{d} \sigma .
$$

Let now $\partial$ stand for any derivative with respect to the $y$ variable. We have

$$
a(v) \cdot \nabla_{y} \partial f-Q(\partial f)=\partial g+\partial Q(f)
$$

where $\partial Q$ is defined like $Q$ but with coefficients $\partial \sigma$ and $\partial \Sigma$. It follows from Proposition 3.1 that $\partial f \in \mathrm{L}^{p}(Y \times V)$. Moreover, since $\partial \sigma \in \mathrm{C}^{0}\left(V, L^{\infty}(Y \times V)\right), \partial \Sigma \in \mathrm{C}^{0}\left(V, L^{\infty}(Y)\right)$, and $f \in \mathrm{C}^{0}\left(V, L^{\infty}(Y)\right)$, it is easy to check that the right-hand side belongs to $\mathrm{C}^{0}\left(V, \mathrm{~L}^{p}(Y)\right)$. It follows from the first part of the proof that $\partial f$ lies in $\mathrm{C}^{0}\left(V, L^{p}(Y)\right)$.

Similar considerations apply to the adjoint problem. Note also that derivation of the equation for the equilibrium function $F$ shows similarly that $F$ belongs to $\mathrm{C}^{0}\left(V, \mathrm{~W}_{\#}^{1, p}(Y)\right), 1 \leq p<\infty$.

Proof of Lemma 3.3. We check that the constant $C$ in Proposition 3.1, which depends on the parameter $x$, is actually locally bounded. Indeed, let $K$ be a compact subset of $\mathbb{R}^{N}$. Let $0<\delta<1$. By using the Heine theorem, we can pick $\eta_{\delta}>0$ such that, for any $x, x^{\prime} \in K$ with $\left|x-x^{\prime}\right| \leq \eta_{\delta}$, we have $\left.\| \sigma(x, \cdot)-\sigma\left(x^{\prime}, \cdot\right)\right) \|_{L^{\infty}(Y \times V \times V)} \leq \delta$. Accordingly $\left\|Q(x)-Q\left(x^{\prime}\right)\right\|_{\mathcal{L}\left(\mathrm{L}_{+}^{p}\right)} \leq \delta$. Then, we can find a finite covering $K \subset \cup_{i=1}^{I \delta} B\left(x_{i}, \eta_{\delta}\right)$. For $x \in K$, we rewrite the equation $\mathcal{T}(x)(f(x))=g(x)$ as

$$
\mathcal{T}\left(x_{i}\right)(f(x))=g(x)+\left(Q(x)-Q\left(x_{i}\right)\right)(f(x)) .
$$


It follows that

$$
\|f(x)\|_{\mathrm{L}^{p}(Y \times V)} \leq \frac{C\left(x_{i}\right)}{1-\delta}\|g(x)\|_{\mathrm{L}^{p}(Y \times V)} .
$$

Hence, we can use the constant $C=C(K, \delta)=\max \left\{C\left(x_{i}\right) /(1-\delta), i \in\left\{1, \ldots, I_{\delta}\right\}\right.$.

Let $\delta_{h}$ stand for a differential quotient with respect to $x$. We get

$$
a(v) \cdot \nabla_{y} \delta_{h} f-Q\left(\delta_{h} f\right)=\delta_{h} g+\delta_{h} Q(f) .
$$

The right-hand side is bounded in $\mathrm{L}^{p}(Y \times V)$, so that $\left(\delta_{h} f\right)_{h>0}$ is bounded in $\mathrm{L}^{p}(Y \times V)$. It implies that $x \mapsto f(x, \cdot)$ is differentiable with values in $\mathrm{L}^{p}(Y \times V)$, and we have

$$
a(v) \cdot \nabla_{y} \partial f-Q(\partial f)=\partial g+\partial Q(f) .
$$

We check the continuity of the right-hand side with respect to $x$, and this gives the continuity of $\partial f$. Similar arguments apply to higher derivatives and to the adjoint equation.

The authors wish to thank L.A. Caffarelli and F. Poupaud for many fruitful discussions concerning this work.

\section{REFERENCES}

[1] G. Allaire, Homogenization and two scale convergence. SIAM J. Math. Anal. 23 (1992) 1482-1518.

[2] G. Allaire and G. Bal, Homogenization of the criticality spectral equation in neutron transport. ESAIM: M2AN 33 (1999) 721-746. Announced in Homogénéisation d'une équation spectrale du transport neutronique. CRAS, Vol. 325 (1997) $1043-1048$.

[3] G. Allaire, G. Bal and V. Siess, Homogenization and localization in locally periodic transport. ESAIM: COCV 8 (2002) 1-30.

[4] G. Allaire and Y. Capdeboscq, Homogeneization of a spectral problem for a multigroup neutronic diffusion model. Comput. Methods Appl. Mech. Engrg. 187 (2000) 91-117.

[5] G. Bal, Couplage d'équations et homogénéisation en transport neutronique. Thèse de doctorat de l'Université Paris 6 (1997).

[6] G. Bal, Homogenization of a spectral equation with drift in linear transport. ESAIM: COCV 6 (2001) 613-627.

[7] C. Bardos, F. Golse and B. Perthame, The Rosseland approximation for the radiative transfer equations. CPAM 40 (1987) 691-721; and CPAM 42 (1989) 891-894.

[8] C. Bardos, F. Golse, B. Perthame and R. Sentis, The nonaccretive radiative transfer equations: Existence of solutions ans Rosseland approximations. J. Funct. Anal. 77 (1988) 434-460.

[9] A. Bensoussan, J.-L. Lions and G. Papanicolaou, Boundary layers and homogenization of transport processes. Publ. Res. Inst. Math. Sci. 15 (1979) 53-157.

[10] H. Brézis, Analyse fonctionnelle, Théorie et applications. Masson (1993).

[11] Y. Capdeboscq, Homogenization of a spectral problem with drift. Proc. Roy. Soc. Edinburgh Sect. A 132 (2002) 567-594; Announced in Homogenization of a diffusion equation with drift. CRAS, Vol. 327 (2000) 807-812.

[12] Y. Capdeboscq, Homogénéisation des modèles de diffusion en neutronique. Thèse Université Paris 6 (1999).

[13] C. Cercignani, The Boltzmann equation and its applications. Springer-Verlag, Appl. Math. Sci. 67 (1988).

[14] F. Chalub, P. Markowich, B. Perthame and C. Schmeiser, Kinetic models for chemotaxis and their drift-diffusion limits. Preprint.

[15] J.-F. Collet, Work in preparation. Personal communication.

[16] R. Dautray and J.-L. Lions, Analyse mathématique et calcul numérique pour les sciences et les techniques, Vol. 3. Masson (1985).

[17] P. Degond, T. Goudon and F. Poupaud, Diffusion limit for non homogeneous and non reversible processes. Indiana Univ. Math. J. 49 (2000) 1175-1198.

[18] R. Di Perna, P.-L. Lions and Y. Meyer, $L^{p}$ regularity of velocity averages. Ann. Inst. H. Poincaré Anal. Non Linéaire 8 (1991) 271-287.

[19] L. Dumas and F. Golse, Homogenization of transport equations. SIAM J. Appl. Math. 60 (2000) 1447-1470.

[20] R. Edwards, Functional analysis, Theory and applications. Dover (1994).

[21] L.C. Evans, The perturbed test function method for viscosity solutions of nonlinear PDE. Proc. Roy. Soc. Edinburgh Sect. A 111 (1989) 359-375.

[22] L.C. Evans, Periodic homogenisation of certain fully nonlinear partial differential equations. Proc. Roy. Soc. Edinburgh Sect. A 120 (1992) 245-265.

[23] P. Gérard and F. Golse, Averaging regularity results for pdes under transversality assumptions. Comm. Pure Appl. Math. 45 (1992) 1-26. 
[24] F. Golse, From kinetic to macroscopic models, in Kinetic equations and asymptotic theory, edited by B. Perthame and L. Desvillettes. Gauthier-Villars, Appl. Math. 4 (2000) 41-121.

[25] F. Golse, P.-L. Lions, B. Perthame and R. Sentis, Regularity of the moments of the solution of a transport equation. J. Funct. Anal. 76 (1988) 110-125.

[26] F. Golse and F. Poupaud, Limite fluide des équations de Boltzmann des semi-conducteurs pour une statistique de Fermi-Dirac. Asymptot. Anal. 6 (1992) 135-160.

[27] T. Goudon and A. Mellet, Diffusion approximation in heterogeneous media. Asymptot. Anal. 28 (2001) 331-358.

[28] T. Goudon and A. Mellet, On fluid limit for the semiconductors Boltzmann equation. J. Differential Equations (to appear).

[29] T. Goudon and F. Poupaud, Approximation by homogeneization and diffusion of kinetic equations. Comm. Partial Differential Equations 26 (2001) 537-569.

[30] T. Goudon and F. Poupaud, Homogenization of transport equations; weak mean field approximation. Preprint.

[31] M. Krein and M. Rutman, Linear operator leaving invariant a cone in a Banach space. AMS Transl. 10 (1962) 199-325.

[32] R. Kubo, H-Theorems for Markoffian Processes, in Perspectives in Statistical Physics, edited by H. Raveché. North Holland (1981).

[33] E. Larsen, Neutron transport and diffusion in heterogeneous media (1). J. Math. Phys. (1975) 1421-1427.

[34] E. Larsen, Neutron transport and diffusion in heterogeneous media (2). Nuclear Sci. Engrg. (1976) 357-368.

[35] E. Larsen and J. Keller, Asymptotic solution of neutron transport processes for small free paths. J. Math. Phys. 15 (1974) 75-81.

[36] E. Larsen and M. Williams, Neutron drift in heterogeneous media. Nuclear Sci. Engrg. 65 (1978) 290-302.

[37] P.-L. Lions and G. Toscani, Diffuse limit for finite velocity Boltzmann kinetic models. Rev. Mat. Ib. 13 (1997) $473-513$.

[38] G. Nguetseng, A general convergence result for a functional related to the theory of homogenization. SIAM J. Math. Anal. 20 (1989) 608-623.

[39] R. Petterson, Existence theorems for the linear, space-inhomogeneous transport equation. IMA J. Appl. Math. 30 (1983) $81-105$.

[40] F. Poupaud, Diffusion approximation of the linear semiconductor Boltzmann equation: Analysis of boundary layers. Asymptot. Anal. 4 (1991) 293-317.

[41] E. Ringeisen and R. Sentis, On the diffusion approximation of a transport process without time scaling. Asymptot. Anal. 5 (1991) 145-159.

[42] L. Tartar, Remarks on homogenization, in Homogenization and effective moduli of material and media. Springer, IMA Vol. in Math. and Appl. (1986) 228-246.

[43] E. Wigner, Nuclear reactor theory. AMS (1961). 